\title{
Algas marinas bentónicas de la costa noroccidental de Guerrero, México
}

\author{
Benthic marine algae of the west coast of Guerrero, Mexico
}

\author{
Luz Elena Mateo-Cid ${ }^{\otimes}$ y A. Catalina Mendoza-González \\ Departamento de Botánica, Escuela Nacional de Ciencias Biológicas, IPN. Carpio y Plan de Ayala, Col. Santo Tomas 11340 México, D.F. \\ \lmateoc@ipn.mx
}

\begin{abstract}
Resumen. Se presentan los resultados de un estudio sobre algas marinas bentónicas en 7 localidades de la costa noroccidental de Guerrero, México. Se determinó la presencia de 163 especies de algas marinas. Se identificaron 17 especies de Cyanobacteria, 93 Rhodophyta, 28 Chlorophyta y 25 de Heterokontophyta. Se citan 54 registros nuevos para el litoral de Guerrero, 2 también nuevos, Myrionema strangulans Greville y Acrochaete ramosa (N.L.Gardner) O'Kelly para la costa del Pacífico. Cada especie se acompaña de datos sobre su distribución en el área de estudio, su estado reproductivo, nivel de marea, hábitat, observaciones, epifitismo y número de herbario o de recolección. Se comparó la riqueza específica entre la estación climática de lluvias y la de secas. La división Rhodophyta dominó en términos de diversidad en relación con las 3 divisiones restantes. La ficoflora de la costa noroccidental de Guerrero es de afinidad tropical y más diversa en la época de secas.

Palabras clave: Cyanobacteria, Rhodophyta, Heterokontophyta, Chlorophyta, riqueza específica.

Abstract. We present results on the study on benthic marine algae in 7 localities from the west coast of Guerrero, Mexico. We report 163 species: 17 Cyanobacteria, 93 Rhodophyta, 28 Chlorophyta and 25 Heterokontophyta. Fifty four are new records for Guerrero; while Myrionema strangulans Greville and Acrochaete ramosa (N.L.Gardner) O'Kelly are new to the Pacific coast of Mexico. Each species includes data on its distribution, reproductive stages, tidal level, facies, epiphytism and herbarium's number. Species diversity was compared for 2 different climatic seasons. The Rhodophyta are dominant in terms of diversity in relation to the other groups. The algal flora of the northwest coast of Guerrero is tropical and the greatest diversity was found during dry seasons.
\end{abstract}

Key words: Cyanobacteria, Rhodophyta, Heterokontophyta, Chlorophyta, species diversity.

\section{Introducción}

La ubicación y fisiografía de la costa del Pacífico de México presenta características notables como la diversidad de accidentes geográficos y los parámetros climáticos. En el Pacífico, las regiones mejor conocidas ficoflorísticamente son el golfo de California y el Pacífico de Baja California (Pedroche et al., 2005, 2008; Norris, 2010). En contraste, la región menos estudiada es la tropical, cuyo límite al norte se encuentra en la frontera entre Nayarit y Sinaloa y al sur en Puerto Madero, Chiapas (Serviere Zaragoza et al., 1993). En esta región se localiza la costa Noroccidental de Guerrero, objeto del presente estudio.

Los primeros registros de algas marinas de la costa de Guerrero se encuentran en el trabajo de Taylor (1945). Posteriormente, estos registros se enriquecieron con los trabajos de Dawson $(1949,1954,1960,1961 \mathrm{a}, \mathrm{b}, 1963 \mathrm{a}$, b), Chávez-Barrera (1972, 1980), León-Álvarez y González-González (1993), Mendoza-González y Mateo-Cid

Recibido: 19 octubre 2011; aceptado: 30 julio 2012
(1998), López et al. (2000, 2004), Pedroche et al. (2002) y Candelaria et al. (2006), quienes mencionan 178 especies de algas marinas para la costa noroccidental de Guerrero, en especial de la bahía de Zihuatanejo, la zona mejor estudiada de esa entidad federativa. En el presente trabajo se incorpora una lista florística de 6 localidades poco estudiadas de esta región, además de Zihuatanejo, aportando nuevos registros de algas marinas bentónicas para el lugar, así como su variación estacional en las épocas de lluvias y secas.

\section{Materiales y métodos}

Las muestras ficológicas se obtuvieron mediante 14 muestreos, 7 en la estación climática de lluvias (septiembre de 1994, 1999, 2001 y 2007; octubre de 1998 y 2000; mayo de 2007) y 7 en la de secas (abril de 2008, 2009, 2010 y noviembre de 1996, 1998, 2003 y 2009). Las algas se recolectaron a mano en el nivel intermareal con ayuda de espátulas y navajas de campo, y se fijaron en formaldehido a $4 \%$ en agua de mar. El material fue procesado y 
depositado en el herbario ENCB. Para la descalcificación de taxa de la familia Corallinaceae y Liagoraceae se utilizó $\mathrm{HNO}_{3}$ 0.6 M. La determinación del material ficológico se llevó a cabo empleando los trabajos de Taylor (1945); Dawson (1953a, 1953b, 1954, 1960, 1961a, 1961b, 1962, 1963a y 1963b); Hollenberg (1961); Abbott y Hollenberg (1976); Norris y Johansen (1981); Ávila y Pedroche (2005), Cho et al. (2008), Norris (2010) y Anagnostidis y Komárek (1988). La secuencia de la lista florística sigue el orden propuesto por Pedroche et al. $(2005,2008)$ y Wynne (2011). La actualización nomenclatural se basó en Guiry y Guiry (2011) y Wynne (2011). Cada especie se acompaña con datos sobre distribución en el área de estudio, estado reproductivo, nivel de marea, sustrato, hábitat, epifitismo y número de herbario o de recolección.

Zona de estudio. El estado de Guerrero se localiza en la costa central mexicana del océano Pacífico, entre los $16^{\circ} 18^{\prime}$ y $18^{\circ} 48^{\prime} \mathrm{N}, 98^{\circ} 03^{\prime}$ y $102^{\circ} 12^{\prime} \mathrm{O}$ (Fig. 1 ). La costa del estado forma parte del Pacífico tropical mexicano (PTM), tiene una longitud de $500 \mathrm{~km}$. que representan el 5.9\% de línea costera total del país. Guerrero posee 3 regiones hidrológicas: Balsas, Costa Grande y Costa Chica-Río Verde, las cuencas más importantes son las del los ríos Balsas-Mezcala, Atoyac, Ixtapa y Nexpa (Perevochtchikova y García-Jiménez, 2006). El clima del área corresponde al subtipo Aw o (W) i, es decir, cálido subhúmedo con 2 periodos de lluvia separados por sequías intraestivales; las lluvias de verano son abundantes, las invernales escasas (menos del 5\% del total anual). La temperatura es relativamente alta (mínima invernal de $18^{\circ} \mathrm{C}$ ). La precipitación pluvial anual es de $1103 \mathrm{~mm}$ (García, 1980). La estación de lluvias se presenta de mayo a octubre y la de secas de noviembre a abril. Las mareas son semidiurnas, con una amplitud de alrededor de 2 metros.

A continuación se describen las 7 localidades donde se obtuvo el material biológico para este estudio (Fig. 1):

Puerto Vicente Guerrero o Playa Escondida. Es una playa en la cual se encuentran escolleras y zonas con pequeños agregados de manglar y zonas arenosas con guijarros y cantos rodados. La recolección se realizó en la zona intermareal y hasta 1 metro de profundidad.

La Barrita. Playa mixta, de pendiente pronunciada, arena gruesa y formaciones rocosas en los extremos, donde existen canales de corriente y pozas intermareales. Aquí se recolectó en la zona intermareal.

Barra de Potosí. Es una playa arenosa con numerosas rocas esparcidas en una área de aproximadamente $900 \mathrm{~m}$; en la zona protegida se encuentra la laguna de Potosí cuyo sustrato es arenoso con restos de moluscos y pequeñas poblaciones de Rhizophora sp. En este margen, el material biológico se obtuvo en la zona intermareal.

Bahía de Zihuatanejo (playas Las Gatas, La Madera y La Ropa). Las Gatas está situada en el sur de la bahía de
Zihuatanejo, es de arena gruesa, limitada en uno de sus extremos por manglar y en el otro por un acantilado de roca ígnea. La Madera está protegida del efecto directo del oleaje por su posición, ya que éste se refracta al entrar a la bahía. La playa es de cantos rodados con una longitud de $75 \mathrm{~m}$, limitada al oeste por una pared de rocas ígneas y al este por otra de rocas metamórficas. La Ropa, es una playa arenosa ubicada al este del puerto de Zihuatanejo, tiene a ambos lados grandes agregados rocosos expuestos al oleaje; en este sitio se recolectó material en la zona intermareal. En esta zona se recolectó en la zona intermareal y hasta $3 \mathrm{~m}$ de profundidad.

Isla Grande o Ixtapa. En esta localidad existe una gran cantidad de material particulado fino sobre las rocas, cabezos de coral (la mayoría fracturados), esqueletos de coral, el fondo es de cantos rodados y guijarros. En esta zona se recolectó en la zona intermareal y hasta $4 \mathrm{~m}$ de profundidad.

Playa Los Troncones-La Majahua. Localidad donde se realizó un recorrido de aproximadamente 2 kilómetros, encontrándose playas arenosas expuestas a fuerte oleaje con numerosos agregados rocosos, los que en ocasiones forman pozas intermareales y canales de corriente. En este lugar se recolectó en la zona intermareal y en pozas intermareales.

Las Peñitas. Playa que se encuentra en la bahía de Petacalco y está protegida del oleaje, la región litoral es mixta, encontrándose playas arenosas con acantilados y agregados rocosos, donde se forman pozas intermareales. En este lugar, el material ficológico se obtuvo en la zona intermareal expuesta.

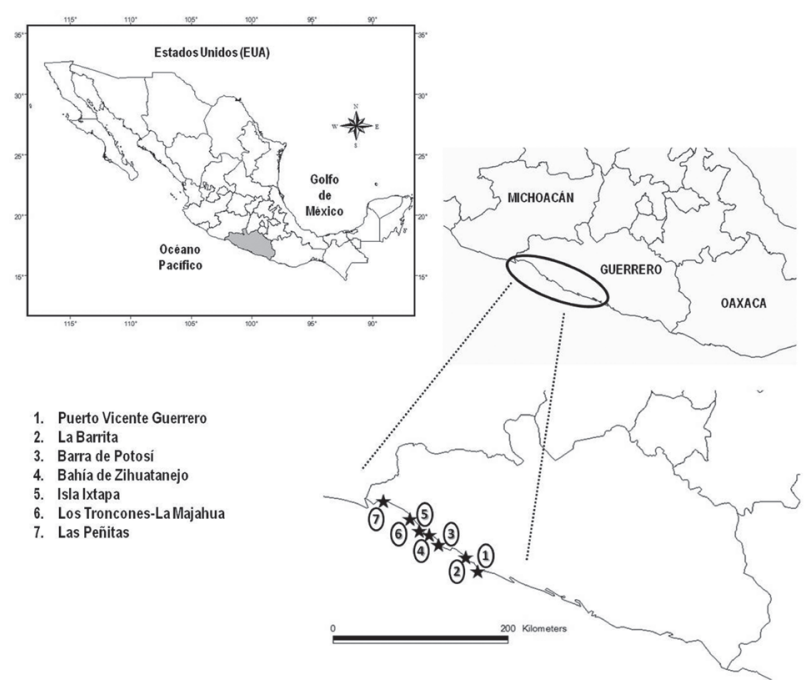

Figura 1. Área de estudio y localidades de muestreo. 


\section{Resultados}

En la costa noroccidental de Guerrero, la cual es predominantemente rocosa, el total de especies de algas marinas bentónicas recolectadas fue de 163; habitan en plataformas rocosas, pozas de marea, sobre guijarros, cantos rodados, zonas de acantilados y canales de corriente. De estas especies, 17 corresponden a Cyanobacteria (10.42\%), 93 a Rhodophyta (57.05\%), 25 a Heterokontophyta (15.33\%) y 28 a Chlorophyta (17.20\%). Los datos indican que la riqueza específica está dominada por las algas rojas, organismos que son importantes por su cobertura y su diversidad en zonas tropicales y templadas; tienen un exitoso desarrollo como epizoicas, epilíticas y epífitas; sin embargo, algunas especies pasan desapercibidas por su escasa talla. En este grupo, las familias mejor representadas fueron: Corallinaceae y Ceramiaceae con 15 especies cada una y Rhodomelaceae con 12 especies (Apéndice). De las Cyanobacteria, que es un grupo con pocos registros previos, fueron localizadas 17 especies; 15 son epífitas, y aun cuando no son un grupo muy diverso y numeroso, su importancia radica en el grado de epifitismo que establecen con sus hospederos, ya que algunas llegan a tener una cobertura hasta del $80 \%$, lo que confiere una coloración verde azul a la superficie de las algas sobre las que se desarrollan.

Para Heterokontophyta se encontraron 25 especies; la familia mejor representada fue Dictyotaceae con 9 taxa, todas de hábito epilítico. En Chlorophyta las familias más importantes fueron Cladophoraceae y Ulvaceae con 7 y 5 especies respectivamente; exceptuando 2 especies de Rhizoclonium que son epifítas, el resto son epilíticas. Como se aprecia en la figura 2, la mayor riqueza específica se encontró en la estación climática de secas con146 taxa y la menor en la de lluvias con 128. En la figura 3, se observa que en las localidades 3, 4, 6 y 7 que corresponden a barra de Potosí, Zihuatanejo, Los Troncones-La Majahua y Las Peñitas, se presentó el número más alto de especies en ambas estaciones climáticas. Los Troncones-La Majahua fue la localidad con mayor riqueza específica de la costa noroccidental de Guerrero con 104 taxa en total, mientras que La Barrita fue la que el menor número de especies tuvo (39; Fig. 3), debido sobre todo a que la playa arenosa presenta una pendiente muy pronunciada, ambiente difícil de colonizar por las algas ya que al accionar el oleaje sobre la arena ésta se convierte en un material móvil y abrasivo que no permite la fijación de esporas o cigotos, por consiguiente, la flora se encontró sobre las rocas de los acantilados.

Asimismo, en este estudio se localizaron principalmente algas anuales, las que dominan en la época de secas y disminuyen en número en la época de lluvias. El epifitismo también fue un factor relevante en este estudio al encontrarse 76 taxa epífitos. Finalmente, la reproducción

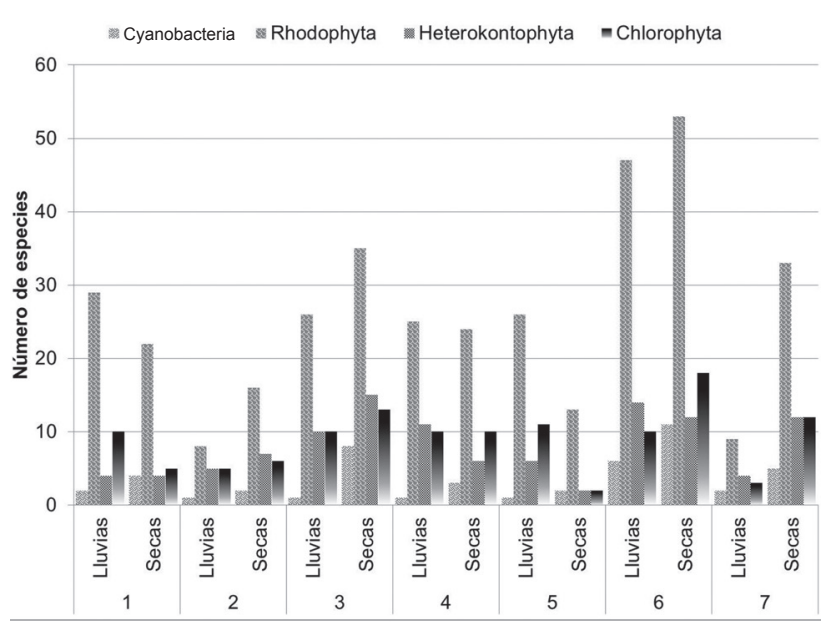

Figura 2. Número de especies por localidad y por estación climática.

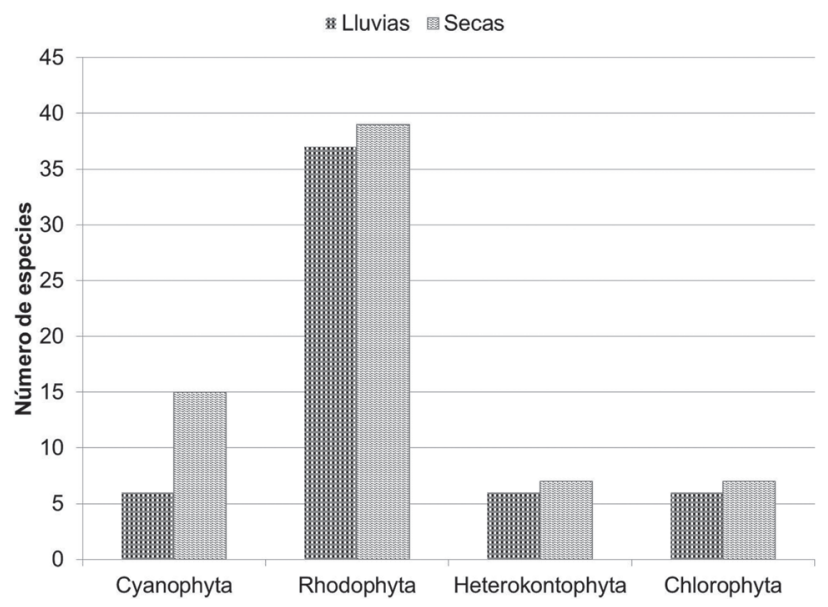

Figura 3. Número total de especies por estación climática.

que predominó en buena parte de los ejemplares ubicados en este estudio fue la asexual.

\section{Discusión}

Biogeografia. Los resultados obtenidos en el presente estudio se analizaron con los índices de Feldmann y Cheney y se compararon con los estudios de Mateo-Cid y Mendoza-González $(1991,1992,2001)$ para Colima, Nayarit y Oaxaca, respectivamente; Mendoza-González y MateoCid (1998) de Guerrero, Dreckmann et al. (2006) para el litoral de Chiapas y Mendoza-González et al. (2011) para la costa sur de Jalisco. Los valores que se obtuvieron con los índices de Feldmann y Cheney para los 6 estados mencionados se muestran en el Cuadro 1; se observa que la ficoflora de la costa noroccidental de Guerrero es similar a la ubicada en Colima, Jalisco y Oaxaca, estados que 
Cuadro 1. Clasificación de la ficoflora con los índices de Feldmann y Cheney

\begin{tabular}{lcc}
\hline Estado & Índice Feldmann $(R / P)$ & Índice de Cheney $(R+C / P)$ \\
\hline Nayarit & 4.31 & 6.12 \\
Colima & 3.35 & 4.52 \\
Oaxaca & 4.31 & 5.0 \\
Costa sur de Jalisco & 3.59 & 4.63 \\
Chiapas & 3.63 & 5.63 \\
Guerrero (este estudio) & 3.72 & 4.84 \\
\hline
\end{tabular}

pertenecen al Pacífico tropical de México; tales valores permiten establecer que la ficoflora de estos estados es mixta, con numerosos elementos tropicales, como Amphiroa beauvoisii J.V. Lamouroux, A, misakiensis Yendo, Neogoniolithon trichotomum (Heydrich) Setchell et L. Mason, Izziella orientalis (J. Agardh) Huisman et Schils, Dictyota crenulata J. Agardh, Asteronema breviarticulata (J. Agardh) Ouriques et Bouzon, Ectocarpus siliculosus (Dillwyn) Lyngbye, Chaetomorpha antennina (Bory de Saint-Vincent) Kützing, Parvocaulis parvulus (SolmsLaubach) S. Berger, U. Fettweiss, S. Gleissberg, L.B. Liddle, U. Richter, H. Sawitzky et G.C. Zuccarello y Ulva lactuca Linnaeus, entre otros. Sin embargo, se encontraron especies de zonas templadas, como Jania ungulata f. brevior (Yendo) Yendo y Mesophyllum crassiusculum (Foslie) P. Lebednik. La ficoflora de Guerrero puede describirse como mixta, debido a que un valor de la relación $\mathrm{R} / \mathrm{P}>4$ se suele encontrar en regiones tropicales, mientras que uno de $\mathrm{R} / \mathrm{P}<2$ corresponde con la ficoflora de regiones templado-frías. De acuerdo con Cheney (1977) los valores de la relación $(\mathrm{R}+\mathrm{C}) / \mathrm{P}>6$ se obtienen en floras tropicales, en tanto que las floras de mares templados-fríos tienen valores $\measuredangle 3$ y como se indica en el Cuadro 1 , los valores de los índices de Feldmann y Cheney para la zona de estudio fueron de 3.72 y 4.84 , respectivamente. Por lo anteriormente mencionado, se establece que la ficoflora de la costa noroccidental de Guerrero es mixta.

Riqueza específica. Como se observa en el Apéndice, de las 163 especies determinadas en este estudio, 53 representan nuevos registros para el área de estudio (NRG); 11 corresponden a Cyanobacteria, grupo de organismos poco estudiados y que en general pasan desapercibidos por su hábito epífito y su tamaño. En el caso de Rhodophyta se encontraron 25 nuevos registros; 11 también son epífitas y de talla pequeña, por lo que es probable que pasen inadvertidas para los recolectores. Para Heterocontophyta y Chlorophyta se ubicaron 8 y 9 registros respectivamente. Las especies Feldmannia simplex (P.L. Crouan et H.M. Crouan) G. Hamel, Hincksia mitchelliae (Harvey) P.C. Silva, Ectocarpus commensalis Setchell et N.L. Gardner, E. siliculosus (Dillwynn) Lyngbye, Cladophoropsis sundanensis Reinbold y Phyllodictyon anastomosans (Harvey)
Kraft et M. J. Wynne, entre otras, pueden clasificarse como algas anuales de acuerdo con su periodo de vida, lo que supone que a menudo sólo viven durante la estación del año que es favorable para su desarrollo. Las varias recolecciones que se realizaron en la costa noroccidental de Guerrero durante este estudio deben considerarse como uno de los motivos por los cuales estas especies pudieron registrarse.

Myrionema strangulans y Acrochaete ramosa representan nuevos registros para el litoral del Pacífico tropical de México (NRM); M. strangulans es epífita y A. ramosa endófita, ambas son microscópicas. Estas especies sólo se localizaron en la estación climática de secas, época en que los hospederos se encuentran en decadencia. Por otro lado, 67 especies registradas previamente en el área de estudio no se localizaron y se encuentran señaladas en el Cuadro 2. Esta situación puede deberse a cambios en los ambientes donde habitan estas algas porque actualmente la influencia humana en estas zonas es mayor, lo que conlleva la modificación del litoral, por la construcción de hoteles, marinas y puertos; conjuntamente con la eventualidad de fenómenos naturales, como los huracanes. También deben considerarse los ciclos de vida de las algas y su estacionalidad. Un punto a considerar es la posibilidad de que registros previos sean nombres mal aplicados, como es el caso de Laurencia intricata J.V. Lamouroux y Gracilaria cervicornis (Turner) J. Agardh, especies privativas del Atlántico de América; asimismo, han sido citadas varias especies de Amphiroa: A. annulata M. Lemoine, A. crosslandii M. Lemoine, A. dimorpha M. Lemoine, A. subcylindrica E.Y. Dawson y A. valonioides Yendo. En este sentido, es bien conocida la problemática que presenta este género en la delimitación de sus especies debida a su plasticidad morfológica, lo que ha derivado en la confusión de nombres y en una sobrestimación del número de especies (RiosmenaRodríguez y Siqueiros-Beltrones, 1996). En este mismo contexto, pueden mencionarse las coralinas incrustantes como Lithophyllum proboscideum (Foslie) Foslie y Lithothamnion validum (Foslie) Foslie, entre otras, ya que en general los géneros de este grupo de organismos presentan un gran problema desde el punto de vista taxonómico por la sobreposición de características que son utilizadas 
Cuadro 2. Registros anteriores de algas marinas para el litoral de Guerrero no ubicados en este estudio

Especie Referencia

Cyanobacteria

1. Oscillatoria margaritifera (Kützing) Gomont

\section{Rhodophyta}

2. Amphiroa annulata M. Lemoine

3. Amphiroa crosslandii M. Lemoine

4.. Amphiroa dimorpha M. Lemoine

5. Amphiroa subcylindrica E.Y. Dawson

6. Amphiroa valonioides Yendo

7. Jania adhaerens J.V. Lamouroux

8. Jania capillacea Harvey

9. Jania pumila J.V. Lamouroux

10. Jania rubens (Linnaeus) J.V. Lamouroux

11. Lithothamnion australe Foslie ( como Lithophyllum australe (Foslie) M. Lemoine)

12. Lithophyllum hancockii E.Y. Dawson

13. Lithophyllum imitans Foslie

14. Lithophyllum proboscideum (Foslie) Foslie

15.Lithothamnion australe Foslie

16. Spongites fruticulosa Kützing (como Lithothamnion fruticulosum (Kützing) Foslie)

17. Lithothamnion phymatodeum Foslie (como Lithothamnion pacificum (Foslie) Foslie)

18.Lithothamnion validum (Foslie) Foslie

19 Haloplegma mexicanum W.R. Taylor

20. Dasya baillouviana (S.G. Gmelin) Montagne (como Dasya pedicellata (C. Agardh) C. Agardh)

21. Callithamnion paschale Børgesen

22.Centroceras clavulatum (C.Agardh) Montagne

23 Ceramium mazatlanense E.Y. Dawson

24 Ceramium paniculatum Okamura

25. Ceramium vagans P.C. Silva

26 Alsidium pusillum E. Y. Dawson

27. Laurencia clarionensis Setchell et N.L. Gardner

28. Laurencia intricata J.V. Lamouroux

29. Laurencia voragina W.R. Taylor

30. Neosiphonia beaudettei (Hollenberg) M. S. Kim et I.A. Abbott (como Polysiphonia beaudettei Hollenberg)

31 Polysiphonia hendryi N.L. Gardner

32 Polysiphonia homoia Setchell et N.L. Gardner
Chávez-Barrera (1972)

Chávez-Barrera (1972)

Salcedo-Martínez et al. (1988)

Candelaria et al. (2006)

Salcedo-Martínez et al. (1988)

López et al. (2004)

Mendoza-González y Mateo-Cid (1998)

Chávez-Barrera (1972), Salcedo-Martínez et al. (1988)

Chávez-Barrera (1972)

Chávez-Barrera (1972)

Chávez-Barrera (1972)

Dawson (1960), Chávez-Barrera (1972), Salcedo-Martínez et al. (1988)

Chávez-Barrera (1972)

Dawson (1960)

Dawson (1960)

Dawson (1960)

Dawson (1960)

Taylor (1945)

Salcedo-Martínez et al. (1988)

Salcedo-Martínez et al. (1988)

López et al. (2004)

Dawson (1962), Chávez-Barrera (1972), Candelaria et al. (2006)

López et al. (2004)

López et al. (2004)

López et al. (2004)

Chávez-Barrera (1972), Salcedo-Martínez et al. (1988)

López et al. (2004)

Taylor (1945), Chávez-Barrera (1972)

Taylor (1945), Salcedo-Martínez et al. (1988)

Hollenberg (1961)

Salcedo-Martínez et al. (1988)

Salcedo-Martínez et al. (1988) 
Cuadro 2. Continúa

Especie Referencia

33 Polysiphonia mollis J.D. Hooker et Harvey

34 Ophidocladus simpliciusculus (P.L. Crouan et H.M. Crouan) Falkenberg (como Rhodosiphonia californica Hollenberg)

35 Gelidium mcnabbianum (E.Y. Dawson) Santelices

36 Gelidium sclerophyllum W.R. Taylor

37 Pterocladiella caloglossoides (M. Howe) Santelices

38 Gelidiella acerosa (Forsskål) Feldmann et G.Hamel

39 Wurdemannia miniata (Sprengel) Feldmann et G. Hamel

40 Ahnfeltiopsis gigartinoides (J. Agardh) P.C. Silva et DeCew

41 Peyssonnelia dawsonii Denizot (como Ethelia mexicana E.Y. Dawson)

42 Grateloupia howeii Setchell et N.L.Gardner

43 Grateloupia prolongata J. Agardh

44 Gracilaria cervicornis (Turner) J. Agardh

45 Gracilaria gracilis (Stackhouse) M. Steentoft, L.M. Irvine et W.F. Farnham (como Gracilaria confervoides (Linnaeus) Greville)

46 Gracilaria textorii (Suringar) De Toni (como Gracilaria vivesii $\mathrm{M}$. Howe)

47 Hypnea valentiae (Turner) Montagne (como Hypnea californica Kylin)

\section{Heterokontophyta}

48 Stragularia clavata (Harvey) G. Hamel

49 Hapalospongidion gelatinosum De A.Saunders

50 Petroderma maculiforme (Wollny) Kuckuck

51 Pseudolithoderma nigrum Hollenberg

52 Ralfsia pacifica Hollenberg

53 Dictyopteris delicatula J. V. Lamouroux

54 Padina concrescens Thivy

55 Colpomenia ramosa W. R. Taylor

56 Rosenvingea intricata (J. Agardh) Børgesen

\section{Chlorophyta}

57 Ulva lobata (Kützing) Harvey

58 Ulva californica Wille

59 Chaetomorpha aerea (Dillwyn) Kützing

60 Cladophora microcladiodes F.S. Collins

61 Phyllodictyon robustum (Setchell et N.L. Gardner) Leliaert et Wysor (como Struveopsis robusta (Setchell et N.L.Gardner) Rhyne et H.Robinson)
Chávez-Barrera (1972), López et al. (2004)

Dawson (1963a)

López et al. (2004)

Dawson (1953), Candelaria et al. (2006)

López et al. (2004)

Dawson (1953)

Dawson (1953), Chávez-Barrera (1972), Salcedo-Martínez et al. (1988)

Candelaria et al. (2006)

Dawson (1953)

Salcedo-Martínez et al. (1988), Mendoza-González y MateoCid (1998)

López et al. (2004)

Chávez-Barrera (1972), Salcedo-Martínez et al. (1988)

Salcedo-Martínez et al. (1988)

Salcedo-Martínez et al. (1988)

Dawson (1961); Taylor (1945)

León-Álvarez y González-González (1993)

León-Álvarez y González-González (1993), Candelaria et al. (2006)

León-Álvarez y González-González (1993)

León-Álvarez y González-González (1993)

León-Álvarez y González-González (1993)

López et al. (2004)

Salcedo-Martínez et al. (1988)

Dawson (1949)

Dawson (1949)

Taylor (1945)

Candelaria et al. (2006)

Chávez-Barrera (1972)

López et al. (2000)

Chávez-Barrera (1972) 
Cuadro 2. Continúa

\begin{tabular}{lr} 
Especie & Referencia \\
\hline 62 Derbesia vaucheriiformis (Harvey) J.Agardh & Chávez-Barrera (1972) \\
63 Bryopsis galapagensis W.R. Taylor & Chávez-Barrera (1972) \\
64 Bryopsis pennata J. V. Lamouroux & Chávez-Barrera (1972) \\
65 Codium brandegeei Setchell et N.L. Gardner & Salcedo-Martínez et al. (1988) \\
66 Codium picturatum Pedroche et P. Silva & Pedroche et al. (2002) \\
67 Codium simulans Setchell et N.L. Gardner & Chávez-Barrera (1972)
\end{tabular}

para separar las especies de los géneros Lithophyllum y Lithothamnion.

Finalmente, existe la posibilidad de que algunas especies hayan sido excluidas por el tipo de muestreo utilizado. En conclusión y considerando los trabajos realizados previamente por Chávez-Barrera (1972, 1980), SalcedoMartínez et al. (1988), León-Álvarez y González-González (1993), Mendoza-González y Mateo-Cid (1998), López et al. (2000, 2004), Pedroche et al. (2002) y Candelaria et al. (2006), así como el presente estudio, tenemos un total de 230 especies conocidas para la costa noroccidental de Guerrero.

Composición. Las condiciones ecológicas indudablemente tienen influencia en la biología y estructura de las algas marinas, dichas condiciones influyen en su presencia o ausencia en una estación en particular. Feldmann (1937) propuso una clasificación de tipos biológicos para las algas caracterizados por su tiempo de vida: 1) las algas anuales, que sólo viven en una estación o en la mayoría de un año y 2) algas perennes, que son capaces de vivir varios años. En este estudio se localizaron principalmente algas anuales, como es el caso de los representantes de las familias Colaconemataceae, Liagoraceae, Ceramiaceae, Rhodomelaceae, Dasyaceae, Delesseriaceae, Acinetosporaceae, Ectocarpaceae, Dictyotaceae, Ulvaceae, Cladophoraceae y Codiaceae. En contraste, las algas perennes están representadas por las familias Corallinaceae, Hapalidiaceae, Ralfsiaceae y Sargassaceae (Apéndice).

Como puede observarse en el Apéndice, las algas anuales dominan en la época de secas y disminuyen en número en la época de lluvias. Asimismo, existe una marcada diferencia entre el numero de taxa por localidades y por estación climática (Fig. 2). Así, en el conjunto de Cyanobacteria hay 10 que son exclusivas de la época de secas, como Chamaecalyx fucicola (Saunders) Komárek et Anagnostidis y Mastigocoleus testarum Lagerheim ex Bornet et Falhault, que son registros nuevos para la costa de Guerrero. Rhodophyta tiene un comportamiento similar, con 14 especies exclusivamente en secas y 11 en lluvias. Por lo que respecta a Heterokontophyta, se hallaron 4 especies en la época de secas y 3 en la de lluvias, y finalmente, para Chlorophyta, se registraron 4 especies exclusivas para secas y sólo 1 en lluvias.

Ejemplos de especies exclusivas por estación del año son: Trichocoleus tenerrimus (Gomont) Anagnostidis, Cyanocystis olivacea (Reinsch) Komárek et Anagnostidis, Xenoccocus pyriformis Setchell et N.L. Gardner, Jania ungulata f. brevior, Ceramium monacanthum J. Agardh, Heterosiphonia crispella (C. Agardh) M.J. Wynne, Chondria repens Børgesen, Laurencia hancockii E.Y. Dawson, Grateloupia doryphora (Montagne) M.A. Howe, Vaucheria velutina C. Agardh, Ectocarpus commensalis Setchell et N. L. Gardner, Myrionema strangulans, Acrochaete ramosa (N. L. Gardner) O'Kelly y Cladophora albida (Nees) Kützing. Estos taxa están en la categoría de algas anuales. En cuanto a las perennes, las que siguen pueden nombrarse como exclusivas: Leptophytum microsporum (Foslie) A. Athanasiadis y W. H. Adey, Phymatolithon lenormandii (J. E. Areschoug) W.H. Adey, Mesophyllum crassiusculum, Hydrolithon samoënse (Foslie) Keats et Y.M. Chamberlain y Amphiroa misakiensis Yendo.

En la figura 2 se observa que en las localidades de Barra de Potosí, Zihuatanejo, Los Troncones-La Majahua y Las Peñitas es donde se ubicó el número más alto de especies en ambas estaciones climáticas, estas 4 localidades son playas donde domina el sustrato rocoso y por ende, comparten entre ellas numerosas especies (Apéndice).

Finalmente, Amphiroa beauvoisii, A. misakiensis, Hypnea pannosa, Gymnogongrus johnstonii, Chnoospora minima, Chaetomorpha antennina y Ulva rigida se registraron en 6 de las 7 localidades de muestreo y en ambas estaciones climáticas. Los géneros Amphiroa, Hypnea y Chnoospora son algas perennes que se encuentran durante todo el año. Por lo que respecta a Chaetomorpha, Gymnogongrus y Ulva se ha documentado que los nutrientes y la temperatura superficial del agua tienen un papel importante en su presencia; en el caso de Ulva, su crecimiento se inicia en enero, alcanza su máximo desarrollo entre mayo y junio 
y decae en septiembre y octubre (Pacheco-Ruíz et al., 2002, Águila-Ramírez et al., 2005).

Reproducción. El mayor número de especies en estado reproductivo se localizó en la época de secas, siendo la fase tetraspórica la que superó a las especies con células de resistencia (hormogonios, gonidangios) o de propagación vegetativa (propágulos), lo que permite considerar que probablemente la generación tetraspórica tenga una mayor supervivencia con respecto a otras generaciones y a otros mecanismos de reproducción, como la propagación vegetativa o la apomeiosis (Ardito y Gómez, 2005). La etapa reproductiva dominante en Rhodophyta fue la tetraspórica. Los resultados coinciden con los obtenidos por Mateo-Cid y Mendoza-González (1991, 1992, 2001) y Magalhães-Lucio y de Castro-Nunes y Castro (2002), quienes mencionan que la dominancia de una fase reproductiva puede estar influida por los cambios temporales de factores ambientales locales. Con respecto a Cyanobacteria, el 17.64\% presentó alguna fase reproductiva asexual. En Heterokontophyta, el 96\% de sus representantes se encontró en reproducción. Se observaron estructuras pluriloculares tanto en la época de lluvias como en la de secas en especies como Asteronema breviarticulatum, Ectocarpus siliculosus y Myrionema strangulans, en tanto que Sargassum howellii, S. liebmanii y Padina crispata presentaron oogonios y anteridios. En contraste, en Chlorophyta sólo el 25\% de las especies presentó algún estado reproductivo, principalmente por gametangios, esto puede deberse a que las especies se encontraban en estadios juveniles y a que las etapas sexuales de estos organismos tienen un periodo de vida corto (Santelices, 1977; Littler et al., 1983).

Especies epifitas. El epifitismo puede ser considerado como una importante estrategia ecológica, con el fin de obtener un sustrato en condiciones óptimas de luz y protección adecuada para el establecimiento y desarrollo de estas especies. En adición, el epifitismo es una variable importante en el estudio de la estructura de las comunidades marinas bentónicas, puesto que al realizar un análisis detallado de las epifitas, se incrementa la riqueza específica de algas marinas registrada para determinada región (Montañés et al., 2003, Meneses y Faria, 2008).

En este estudio se observó la relevancia del epifitismo, como se muestra en la figura 4 durante todo el periodo de estudio se presentaron especies epifítas. El mayor número de epífitas se encontró en la estación climática de secas con 68 especies, observándose en este hábitat un incremento en el número de taxa de Cyanobacteria y de las familias Ectocarpaceae y Ceramiaceae las cuales alcanzan una cobertura de hasta el $90 \%$ de la superficie de sus hospederos, cuando éstos se encuentran en decadencia en esta estación climática, a diferencia de la época de lluvias en donde las poblaciones de macroalgas bentónicas se encuentran en florecimiento y sólo se localizaron 55 epífitas. El conjunto de epífitas fueron 76 especies, de las cuales 39 se desarrollan exclusivamente sobre otras algas marinas, lo que se debe a que las epífitas se establecen en una determinada especie de alga con la que están estrechamente relacionadas por la textura de la hospedante. Además, la longevidad del hospedero debe alcanzar un tiempo determinado que permita a la epífita completar su ciclo vital (Santelices, 1977). Del total de epífitas, 15 especies corresponden a Cyanobacteria, 46 a Rhodophyta, 9 a Heterokontophyta y 6 a Chlorophyta. De acuerdo con Montañés et al. (2003), los talos laminares y flabelados como los del género Padina, constituyen un sustrato idóneo para el establecimiento de numerosas epifítas. En este trabajo se encontró que la mayoría de las especies epífitas filamentosas pertenecientes a la familia Ceramiaceae y Rhodomelaceae, frecuentemente se hallaron creciendo sobre Amphiroa beauvoisii J.V. Lamouroux, A. misakiensis y Jania pacifica J.E. Areschoug. También se encontraron numerosas epífitas sobre Chaetomorpha antennina, Sargassum howellii Setchell y S. liebmanii J. Agardh; en este contexto, se ha sugerido que la longevidad del huésped debe ser suficiente para permitir al epífito completar su ciclo vital y que ésta podría ser una razón de la ausencia de epífitas en las algas anuales y efímeras (Santelices, 1977). El elevado número de epífitas que se han encontrado en este estudio también se ha observado en sitios cercanos, como Manzanillo, Puerto Vallarta, la costa sur de Jalisco y Oaxaca (Mateo-Cid y Mendoza-González, 1991, 2001; MendozaGonzález y Mateo-Cid, 1992; Mendoza-González et al., 2011).

El análisis y la integración de los estudios previos realizados en el área de estudio y los resultados obtenidos en este trabajo, nos permiten visualizar la importancia de realizar inventarios ficoflorísticos en el litoral mexicano. Además, este tipo de investigaciones nos permite conocer que aún existen regiones que no están exploradas. Por lo que es necesario incrementar las exploraciones en estas regiones para recolectar material ficológico y concluir con el inventario ficoflorístico, ya que algunas zonas no visitadas, como la de Tlacoyunque a Tierra Colorada, con cualidades para el establecimiento y desarrollo de las algas; también es importante considerar las lagunas costeras que tienen condiciones definidas y que no han sido exploradas. Además, es necesario realizar estudios poblacionales y ecológicos de grupos como las algas Coralinas, de las especies epifítas y de aquellas con interés económico.

\section{Agradecimientos}

Al Instituto Politécnico Nacional por facilitar las instalaciones y equipo necesario para el desarrollo de este estudio. Las autoras agradecen la beca otorgada por la 


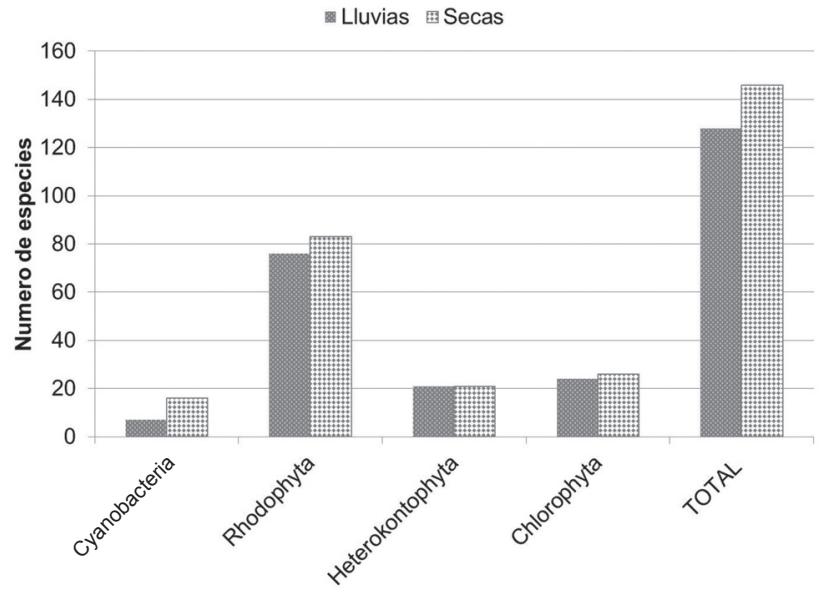

Figura 4. Total de especies epífitas por estación climática.

Comisión de Operación y Fomento de Actividades Académicas (COFAA). La primera autora agradece al programa de Estímulos a la Investigación (EDI) los incentivos recibidos. A Deisy García López y Ulises Sandoval Rauda por el apoyo logístico.

\section{Literatura citada}

Abbott, I. A. y G. J. Hollenberg. 1976. Marine algae of California. Stanford, California. 789 p.

Águila-Ramírez, R. N., M. M. Casas-Valdez, C. J. HernándezGuerrero y A. Marín-Álvarez. 2005. Biomasa de Ulva spp. (Chlorophyta) en tres localidades del malecón de La Paz, Baja California Sur, México. Revista de Biología Marina y Oceanografía 40:55-61.

Anagnostidis, K. y J. Komárek. 1988. Modern approach to the classification system of Cyanophytes. 3. Oscillatoriales. Archv fur Hydrobiologie, Supplement 80:327-472.

Ardito, S. y S. Gómez. 2005. Patrón fenológico de una población de Gelidium serrulatum J. Agardh (Rhodophyta, Gelidiales) en la localidad de Taguao, Estado Vargas, Venezuela. Acta Botanica Venezuelica 28:101-111.

Ávila Ortiz, A. y F. F. Pedroche. 2005. El género Padina (Dictyotaceae, Phaeophyceae) en la región tropical del Pacífico mexicano. In Monografías ficológicas, A. Sentíes G. y K. Dreckmann (eds.). Universidad Autónoma Metropolitana/ Universidad Autónoma de Baja California. México, D. F. p. 139-171.

Báez, J. C., R. Real, J. M. Vargas y A. Flores-Moya. 2004. Revisión crítica de los estudios sobre biogeografía de macroalgas marinas del Mediterráneo. Acta Botánica Malacitana 29:5-11.

Candelaria Silva, C., D. Rodríguez Vargas, N. A. López Gómez y J. González-González. 2006. Patrón de distribución de algas en un canal de corrientes. Revista Especializada en Ciencias
Químico-Biológicas 9:65-72.

Chávez-Barrera, M. L. 1972. Estudio de la flora marina de la bahía de Zihuatanejo y lugares adyacentes. Memorias del IV Congreso Nacional de Oceanografía, 17-19 noviembre1972, México, D. F. Universidad Nacional Autónoma de México, Secretaria de Marina y Secretaria de Educación Pública, México, D. F. p. 265-271.

Chávez-Barrera, M. L. 1980. Distribución del género Padina en las costas de México. Anales Escuela Nacional de Ciencias Biológicas, México 23:45-51.

Cheney, D. P. 1977. A new improved ratio for comparing seaweed floras. Journal of Phycology 13(supplement):1-13.

Cho, T. O., M. B. Sung, M. H. Hommersand, C. A. Maggs, L. McIvor y S. Fredericq. 2008. Gayliella gen. nov. in the Tribe Ceramieaea (Ceramiaceae, Rhodophyta) based on molecular and morphological evidence. Journal of Phycology 44:721738.

Dawson, E. Y. 1949. Resultados preliminares de un reconocimiento de las algas marinas de la costa pacífica de México. Revista de la Sociedad Mexicana de Historia Natural 9:215-255.

Dawson, E. Y. 1953a. Resumen de las investigaciones recientes sobre algas marinas de la costa pacífica de México, con una sinopsis de la literatura, sinonimia y distribución de las especies descritas. Revista de la Sociedad Mexicana de Historia Natural 13:97-197.

Dawson, E. Y. 1953b. Marine red algae of Pacific Mexico I. Bangiales to Corallinoideae. Allan Hancock Pacific Expeditions 17:1-239.

Dawson, E. Y. 1954. Marine red algae of Pacific Mexico II. Cryptonemiales. Allan Hancock Pacific Expeditions 17:241397.

Dawson, E. Y. 1960. Marine red algae of Pacific Mexico III. Cryptonemiales. Corallinaceae, subfamily Melobesioideae. Pacific Naturalist 281:1-125.

Dawson, E. Y. 1961a. A guide to the literature and distribution of Pacific benthic algae from Alaska to the Galapagos Islands. Pacific Science 15:370-461.

Dawson, E. Y. 1961b. Marine red algae of Pacific Mexico. Part 4. Gigartinales. Pacific Naturalist 2:191-341.

Dawson, E. Y. 1962. Marine red algae of Pacific México Part 7. Ceramiales; Ceramiaceae, Delesseriaceae. Allan Hancock Pacific Expeditions 26:1-27.

Dawson, E. Y. 1963a. Marine red algae of Pacific México Part 6. Rhodymeniales. Nova Hedwigia 5:437-476.

Dawson, E. Y. 1963b. Marine red algae of Pacific Mexico. Part 8. Ceramiales: Dasyaceae, Rhodomelaceae. Nova Hedwigia 6:437-476.

Dreckmann, K. M., F. F. Pedroche y A. Sentíes-Granados. 1990. Lista florística de las algas marinas bentónicas de la costa norte de Michoacán, México. Boletín de la Sociedad Botánica de México 50:19-42.

Dreckmann, K. M., A. Sentíes, F. F. Pedroche y M. Callejas. 2006. 
Diagnostico florístico de la ficología marina bentónica en Chiapas. Hidrobiológica 16:147-158.

Feldmann, J. 1937. Recherches sur la vegetation marine de la Mediterranée. La Cote des Alberes. Revue Algologique 10:1339.

García, E. 1980. Modificaciones al sistema de clasificación climática de Köppen (para adaptarlo a las condiciones de la República Mexicana). Instituto de Geografía, Universidad Nacional Autónoma de México, México, D. F. 246 p.

Guiry, M. D y W. D. Guiry. 2011. AlgaeBase version 4.2. World-wide electronic publication, National University of Ireland, Galway. http://www.algaebase.org; última consulta: 10.X.2011.

Hollenberg, G. J. 1961. Marine red algae of Pacific Mexico. The genus Polysiphonia. Pacific Naturalist 2:345-375.

León-Álvarez, D. y J. González-González. 1993. Algas costrosas del Pacífico tropical. In Biodiversidad marina y costera de México, S. I. Salazar Vallejo y N. E. González (eds.). Comisión Nacional para el Conocimiento y Uso de la Biodiversidad/ Centro de Investigaciones de Quintana Roo, México, D. F. p. 456-474.

Littler, M. M., D. S. Littler y P. R. Taylor. 1983. Evolutionary strategies in a tropical barrier reef system:functional form groups of macroalgae. Journal of Phycology 19:229-237.

López, N., D. Rodríguez, C. Candelaria-Silva y J. GonzálezGonzález. 2000. Subtidal macroalgal communities in Acapulco and Zihuatanejo, México. In Aquatic ecosystems of Mexico. Status and scopes, M. Munawar, S. G Lawrence, I. F. Munawar y D. F. Malley (eds.). Backhuys, Leiden. p. 335-352.

López, N., D. Rodríguez y C. Candelaria-Silva. 2004. Intraspecific morphological variation in turf-forming algal species. Universidad y Ciencia. Número Especial 1:7-15.

Magalhães-Lucio, A. y J. M. de Castro-Nunes. 2002. Aportación al conocimiento fenológico de las rodofíceas marinas de la playa del Guarajuba (Camaçari, Bahía) Brasil. Botanica Complutensis 26:17-34.

Mateo-Cid, L. E. y A. C. Mendoza-González. 1991. Algas marinas bénticas de la costa del estado de Colima, México. Acta Botanica Mexicana 13:9-30.

Mateo-Cid, L. E. y A. C. Mendoza-González. 1992. Algas marinas bentónicas de la costa sur de Nayarit, México. Acta Botanica Mexicana 20:13-28.

Mateo-Cid, L. E. y A. C. Mendoza-González. 2002 [2001]. Algas marinas bentónicas de la costa de Oaxaca, México. Anales de la Escuela Nacional de Ciencias Biológicas 47:11-23.

Mendoza-González, A. C. y L. E. Mateo-Cid. 1998. Avance de un estudio sobre las macroalgas marinas de Guerrero y Oaxaca. Ciencia y Mar 4:15-29.

Mendoza-González, A. C., L. E. Mateo-Cid y C. Galicia-García. 2011. Integración florística de las algas marinas de la costa sur de Jalisco, México. Revista Mexicana de Biodiversidad 82:19-49.
Menezes-de Széchy, M. T. y A. D. Faria-de Sá. 2008. Variacão sazonal do epifitismo por macroalgas em una populacão de Sargassum vulgare C. Agardh (Phaeophyceae, Fucales) da Bahia da Ilha Grande, Rio de Janeiro. Oecologia Brasileira 12: 299-314.

Montañés, M. A., J. Reyes y M. Sansón. 2003. La comunidad de epífitos de Zonaria tournefortii en el norte de Tenerife (Islas Canarias); análisis florístico y comentarios sobre su epifauna. Vieraea 31: 121-132.

Norris, J. N. 2010. Marine algae of the northern Gulf of California: Chlorophyta and Phaeophyceae. Smithsonian Contributions to Botany 94:1-276.

Norris, J. N. y H. W. Johansen. 1981. Articulated coralline algae of the Gulf of California, México. I. Amphiroa Lamouroux. Smithsonian Contributions to the Marine Sciences 9:1-29.

Pacheco-Ruiz I., J. A. Zertuche-González, A. Chee-Barragán y E. Arroyo-Ortega. 2002. Biomass and potential commercial utilization of Ulva lactuca (Chlorophyta, Ulvaceae) beds along the North West coast of the Gulf of California. Phycologia 41:199-201.

Pedroche, F. F., P. C. Silva y M. Chacana. 2002. El género Codium (Codiaceae, Chlorophyta) en el Pacífico de México. In Monografías ficológicas, A. Sentíes y K. M. Dreckmann (eds.). Universidad Autónoma Metropolitana- Iztapalapa/ Red Latinoamericana de Botánica, México, D.F. p. 11-74.

Pedroche, F. F., P. C. Silva, L. E. Aguilar-Rosas, K. M. Dreckmann y R. Aguilar-Rosas. 2005. Catálogo de las algas marinas bentónicas del Pacífico de México. I. Chlorophycota. México, D.F. Universidad Autónoma Metropolitana/ Universidad Autónoma de Baja California/ University of California, México, D. F. 135 p.

Pedroche, F. F., P. C. Silva, L. E. Aguilar-Rosas, K. M. Dreckmann y R. Aguilar-Rosas. 2008. Catálogo de las algas marinas bentónicas del Pacífico de México. II. Phaeophycota. México, D.F. Universidad Autónoma Metropolitana/ Universidad Autónoma de Baja California/ University of California, México, D.F. 146 p.

Perevochtchikova, M. y F. García-Jiménez. 2006. Análisis cualitativo de la red hidrométrica actual del estado de Guerrero, México. Investigaciones Geográficas, Universidad Nacional Autónoma de México 61:24-37.

Riosmena-Rodríguez, R. y D. A. Siqueiros-Beltrones. 1996. Taxonomy of the genus Amphiroa (Corallinales, Rhodophyta) in the southern Baja California Peninsula, Mexico. Phycologia 32:135-147.

Salcedo-Martínez, S., G. Green, A. Gamboa y P. Gómez. 1988. Inventario de macroalgas y macroinvertebrados bénticos, presentes en áreas rocosas de la región de Zihuatanejo, Guerrero, México. Anales del Instituto de Ciencias del Mar y Limnologia 15:73-96.

Santelices, B. 1977. Ecología de las algas marinas bentónicas. Universidad Católica de Chile, Santiago de Chile. 384 p. 
Serviere-Zaragoza, E., J. González-González y D. Rodríguez. 1993. Ficoflora de la región de Bahía Banderas, JaliscoNayarit. In Biodiversidad marina y costera de México, S. I. Salazar-Vallejo y N. E. González (eds.). Comisión Nacional para el Conocimiento y Uso de la Biodiversidad y Centro de Investigaciones de Quintana Roo, México. p. 475-485.
Taylor, W. R. 1945. Pacific marine algae of the Allan Hancock Expeditions to the Galapagos Islands Allan Hancock Pacific Expeditions. 12:1-528.

Wynne, M. J. 2011. A checklist of benthic marine algae of the tropical and subtropical western Atlantic: third revision. Nova Hedwigia Beiheft 129:152 p. 


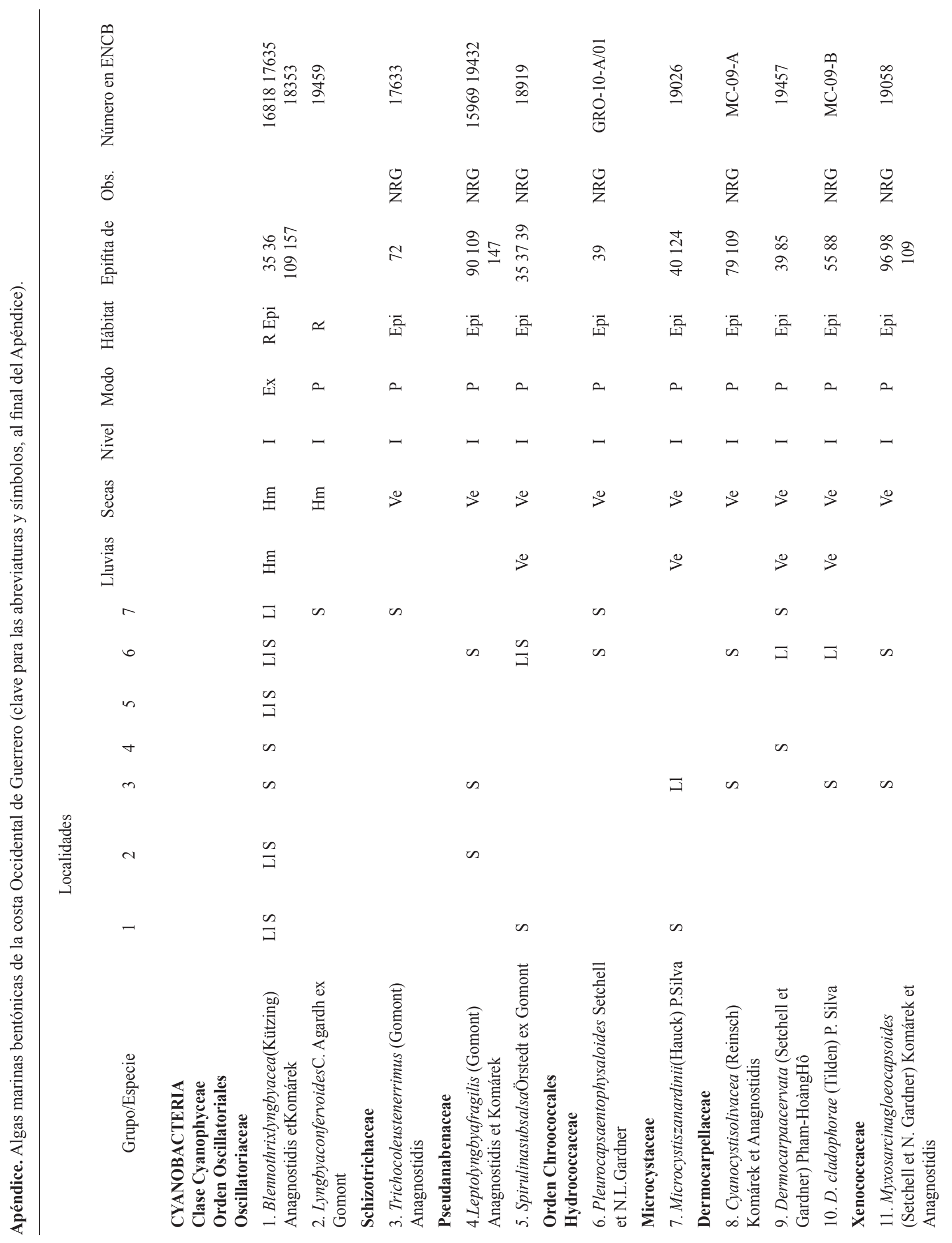




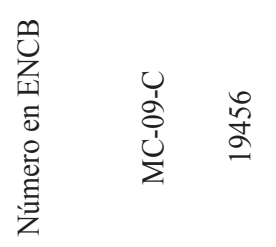

ठั

畜离学

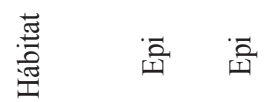

윤 a a

这 -

总 $>$

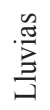

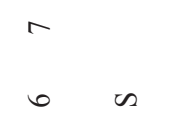

n

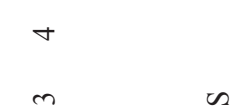

\begin{tabular}{l}
$\frac{0}{0}$ \\
$\frac{0}{0}$ \\
$\frac{\pi}{7}$ \\
0 \\
\hline
\end{tabular}

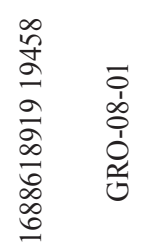

莣

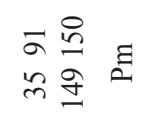

훕 $\simeq$

a. 肴

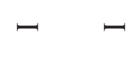

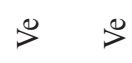

$>$

$\exists \backsim$

$\stackrel{n}{\exists}$

$\exists$ 的

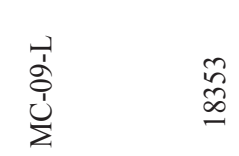

厸

+ $\begin{array}{ll}+ \\ m\end{array}$

훕

通

a

$-$

8

$\infty \omega$



$\infty$

a $\therefore$

$\stackrel{2}{2}$

$\exists$

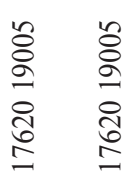

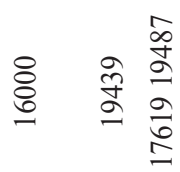

光

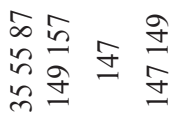

递总畐

a a a

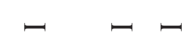

$\therefore \%$

$>$

in as

ヨ

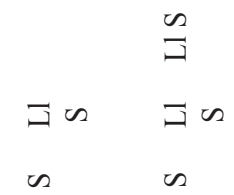

a n

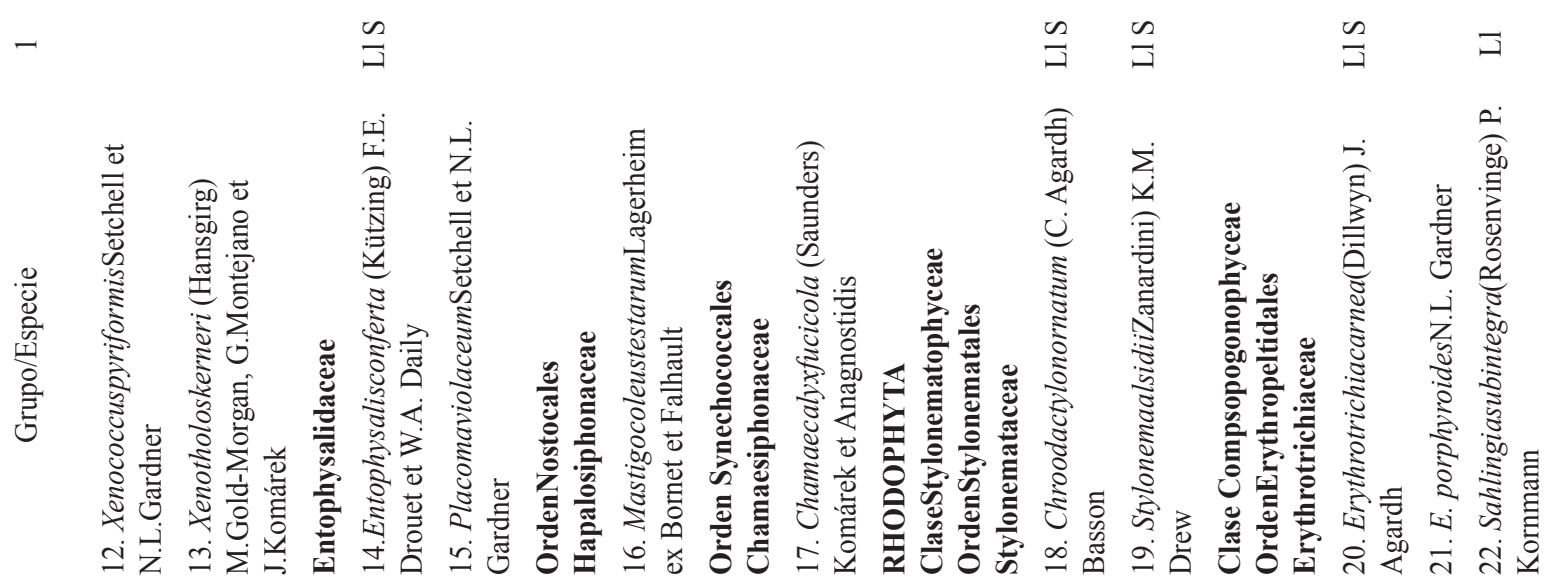




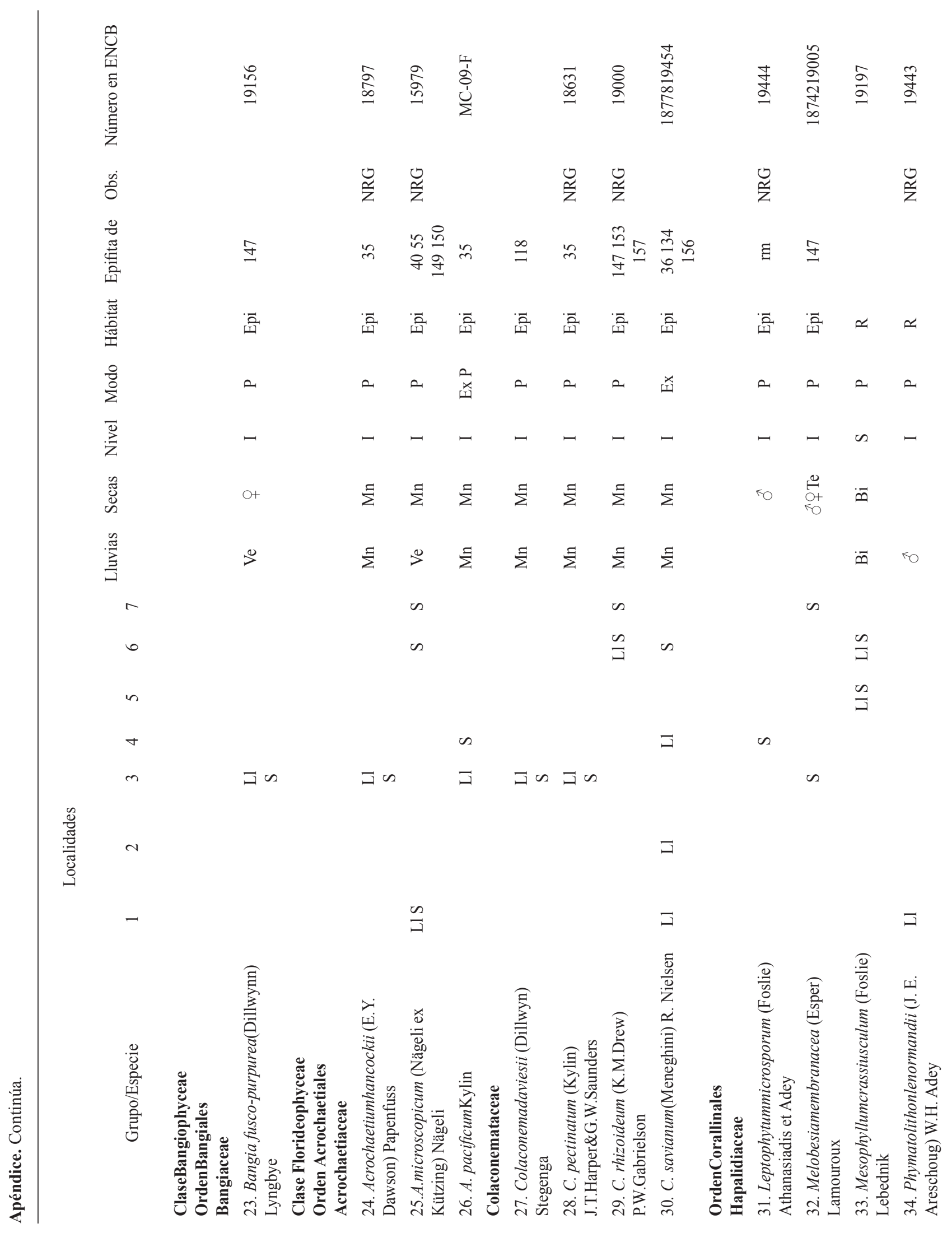




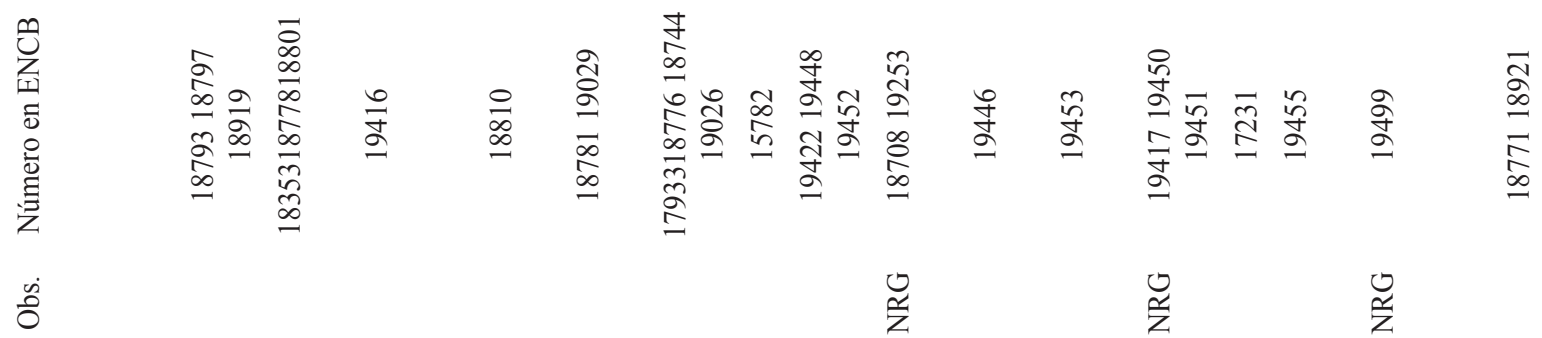

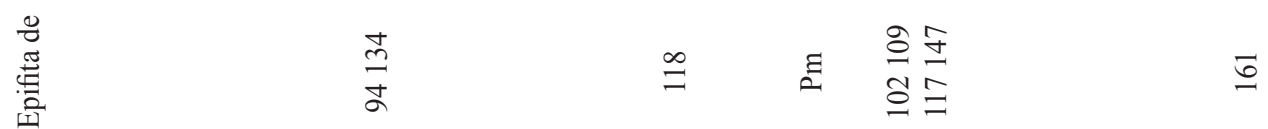

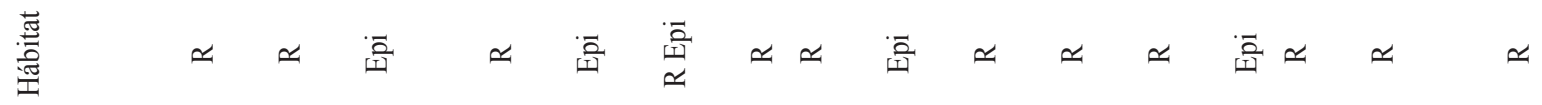

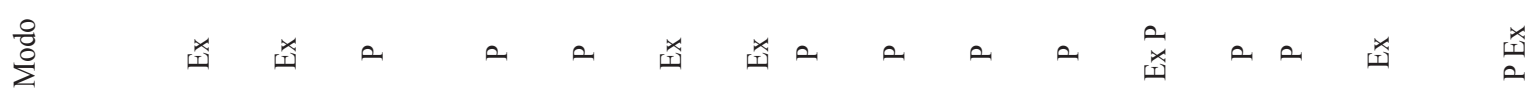

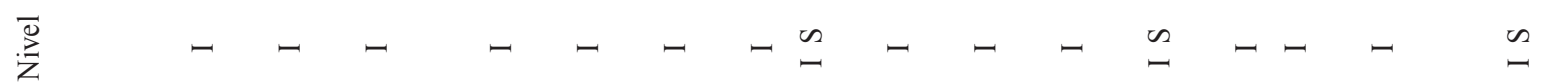

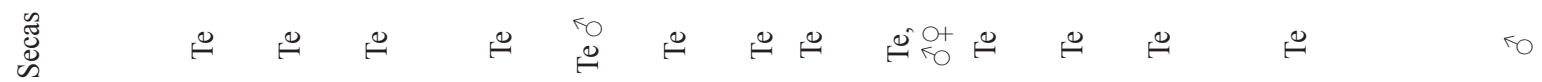

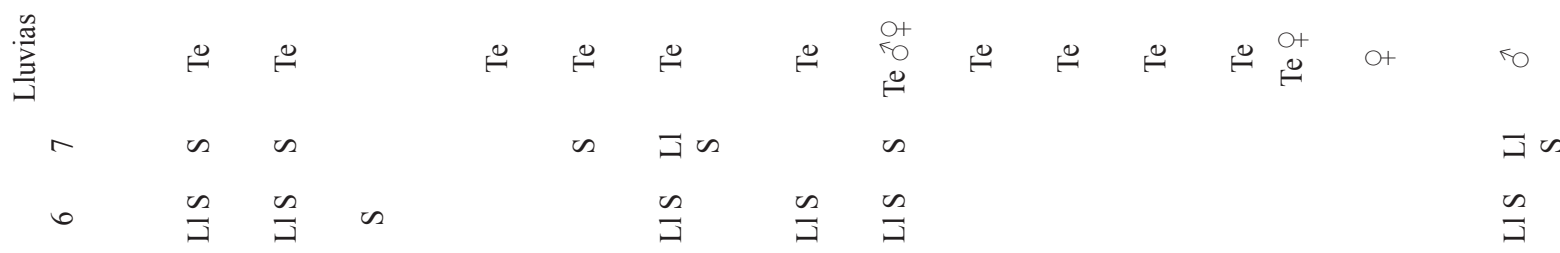

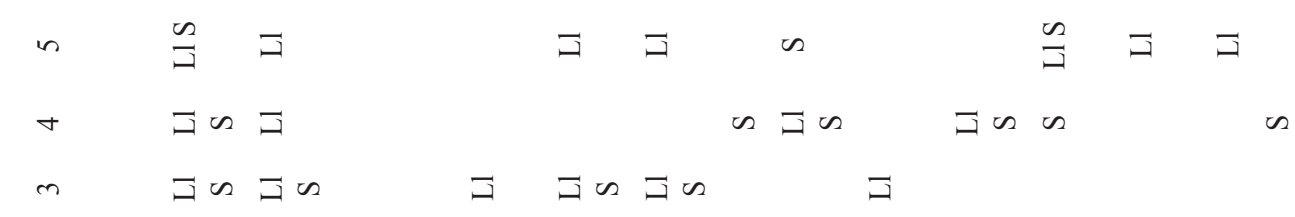
茑

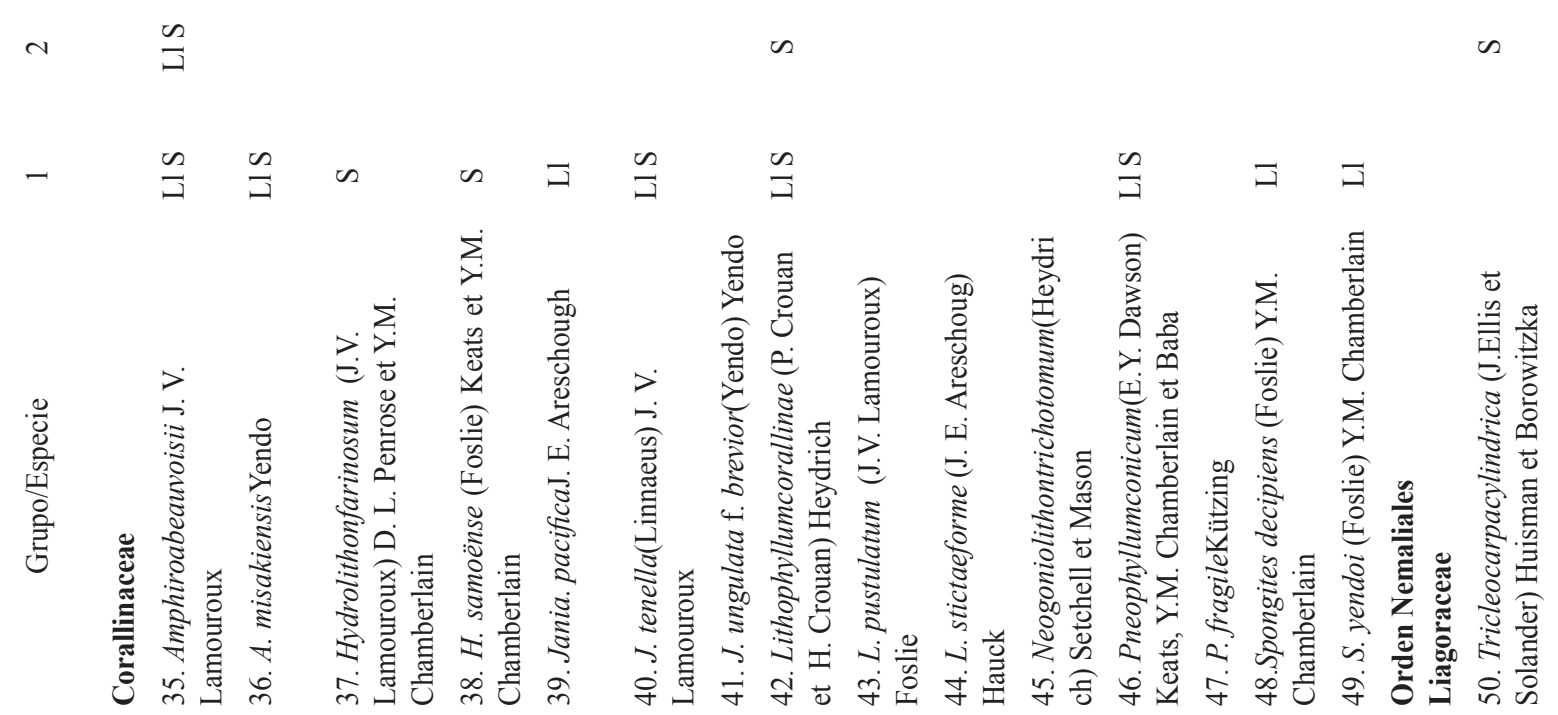




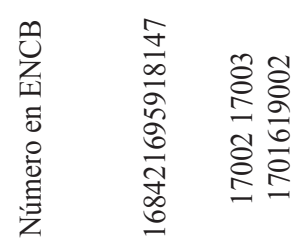

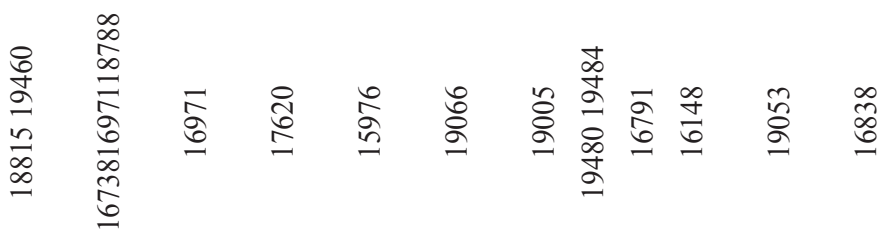

$\stackrel{0}{0}$

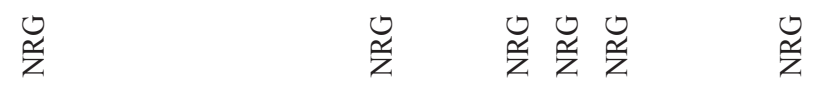

苗

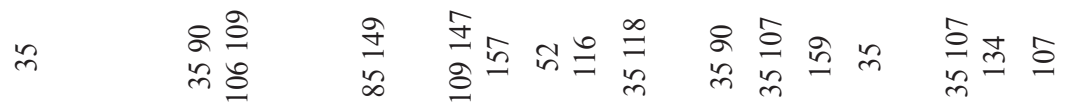

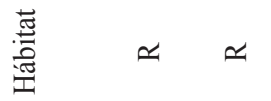

产

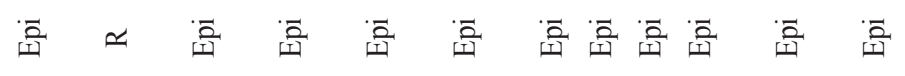

总 希希

$a$

2 a a a a

and a d 肴

吝 -

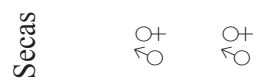

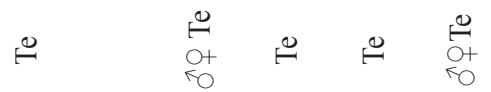

$\oplus>\oplus \oplus \oplus \stackrel{\oplus}{\oplus}$

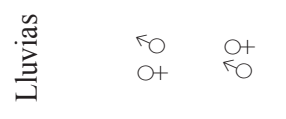

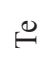

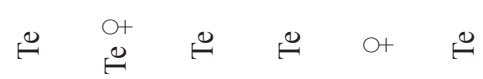

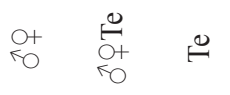

○业当舟

a

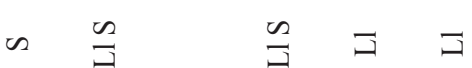

ב

in

$\checkmark \quad \exists \backsim$

m

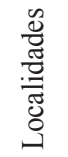

N

$-\quad \cong$

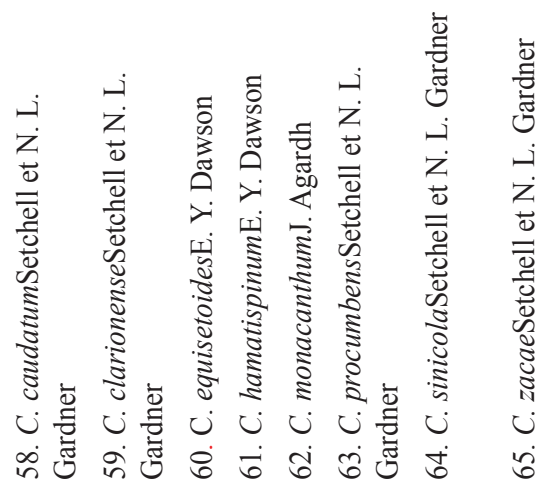




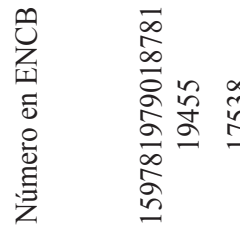

ठิ

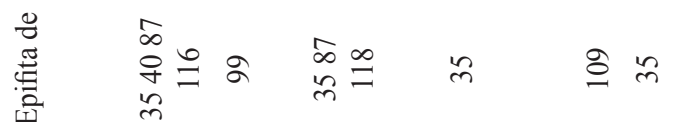

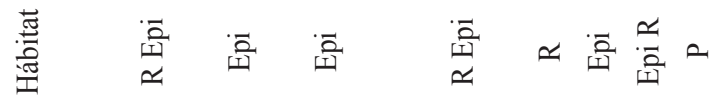

$\frac{8}{8}$

$$
\text { 高 }
$$

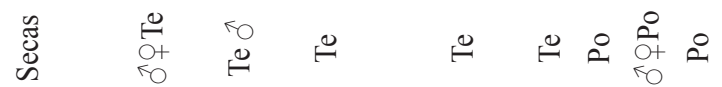

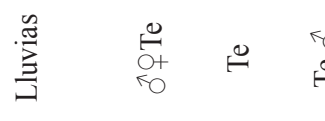

$$
n \text { an as as as as }
$$

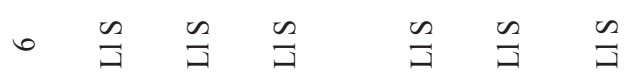$$
\text { in } \exists
$$$$
\text { ナコッ コッ }
$$$$
\text { m } \backsim
$$$$
\text { ヨココ コ }
$$$$
\text { ヨn }
$$

岂

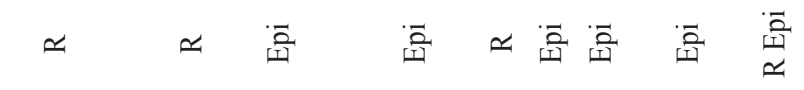

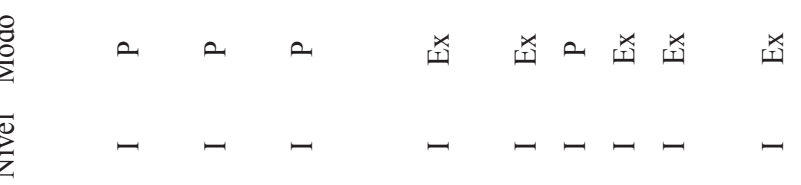

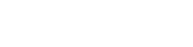

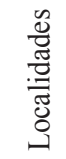

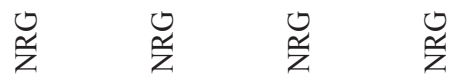

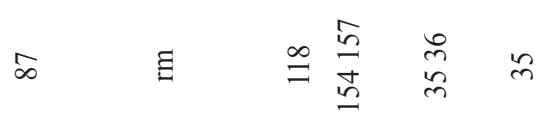

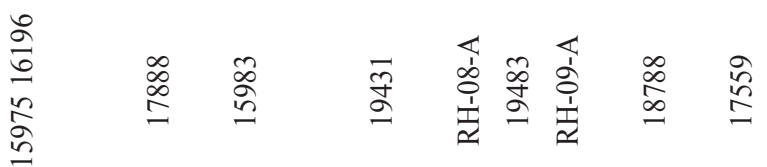
.

㞌㞌 $-\quad-$

$\stackrel{\oplus}{\oplus}$

$\Leftarrow \oplus \stackrel{\oplus}{\oplus} \oplus$

ニ

$\infty \stackrel{\sim}{コ}$ 事 $2+2 a 2$

$r$ is is is

-2 J

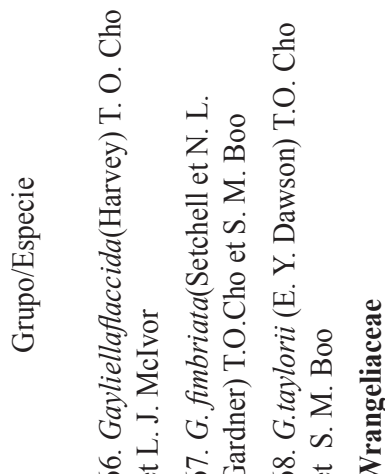

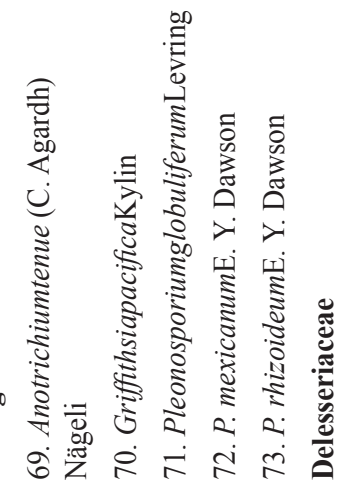

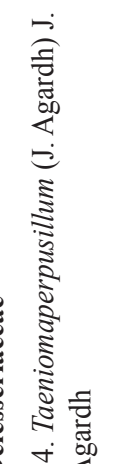<smiles>C=[SiH]</smiles>

ஏ
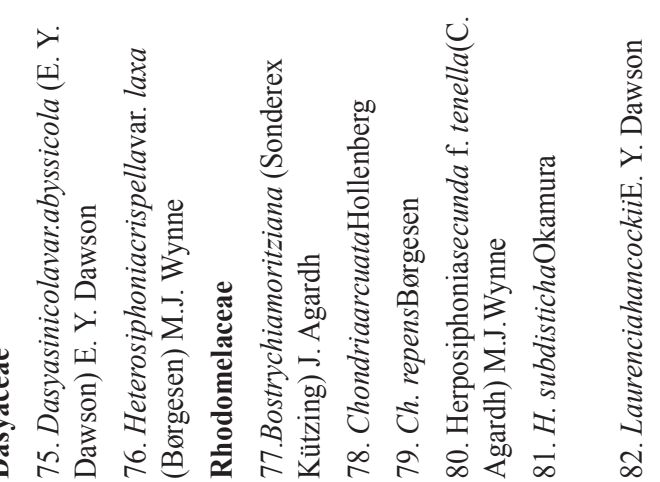


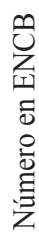

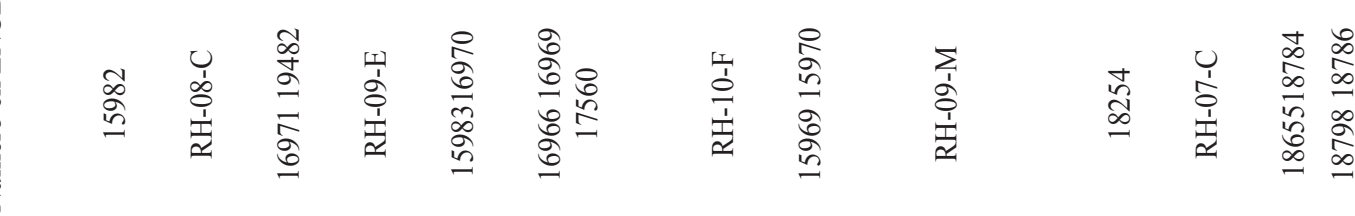

芒

产

苞

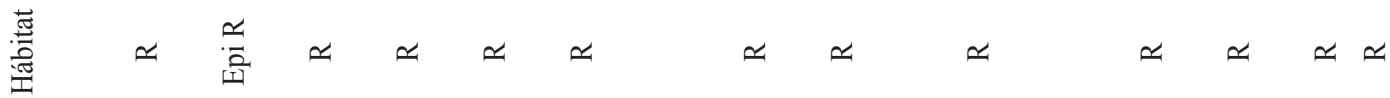

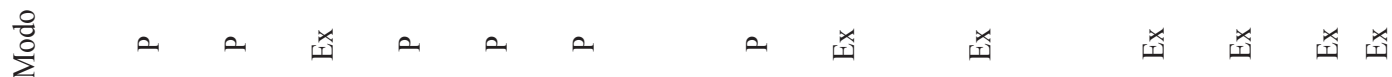

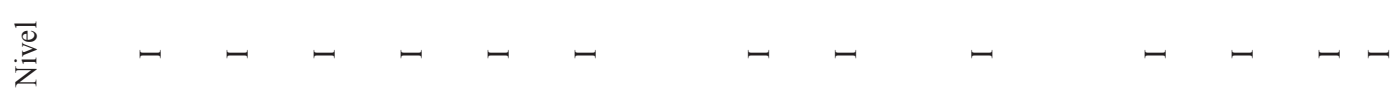

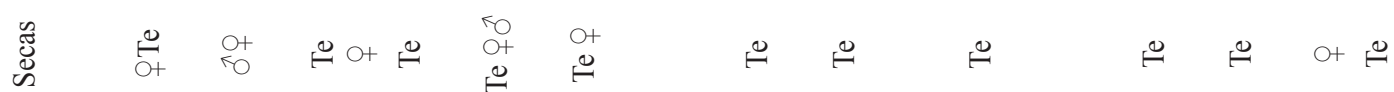

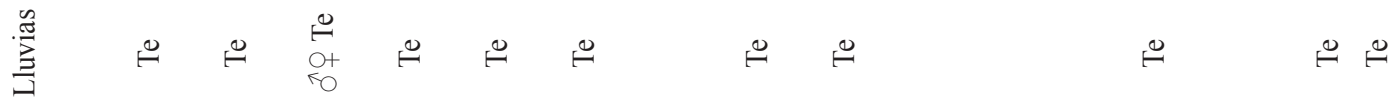

ー ヨथ

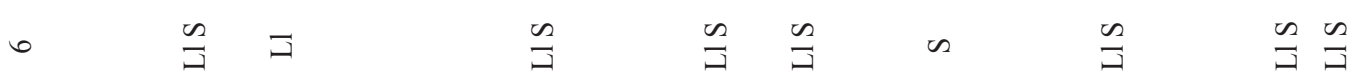

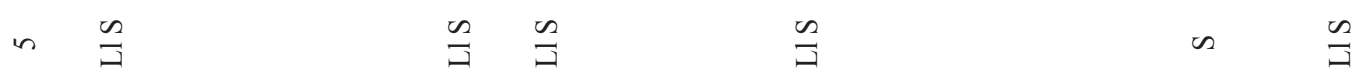

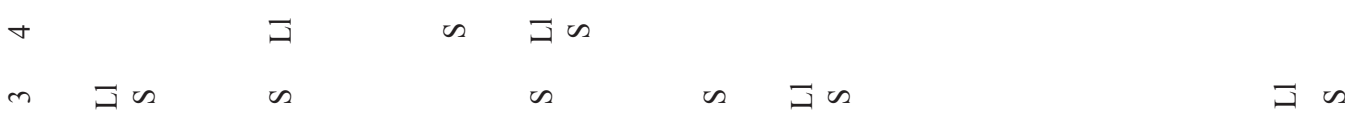

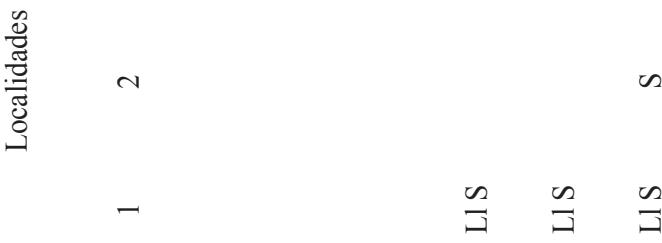

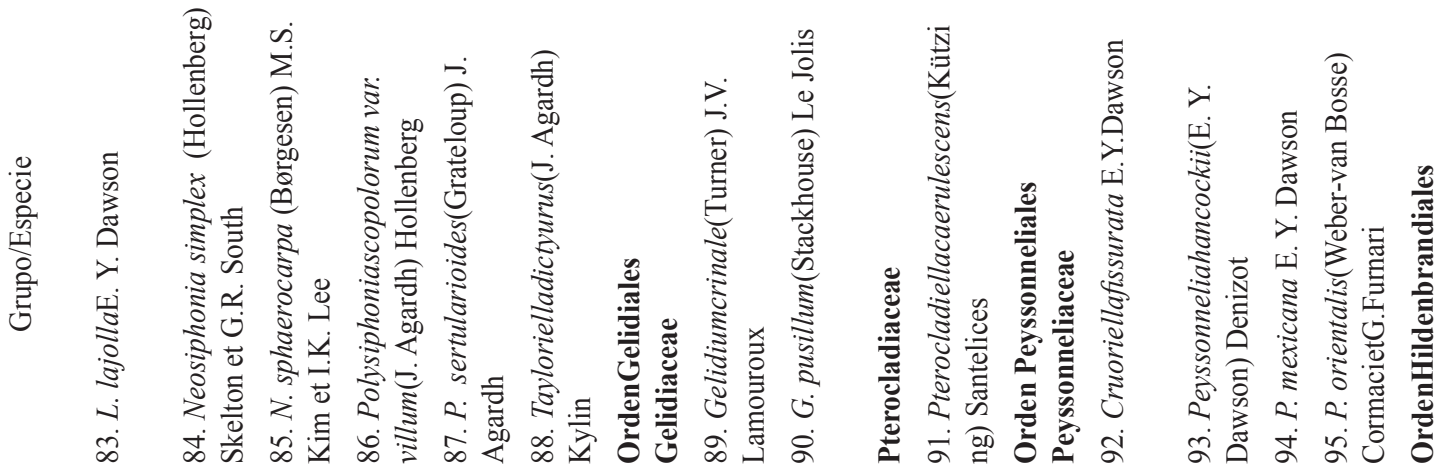




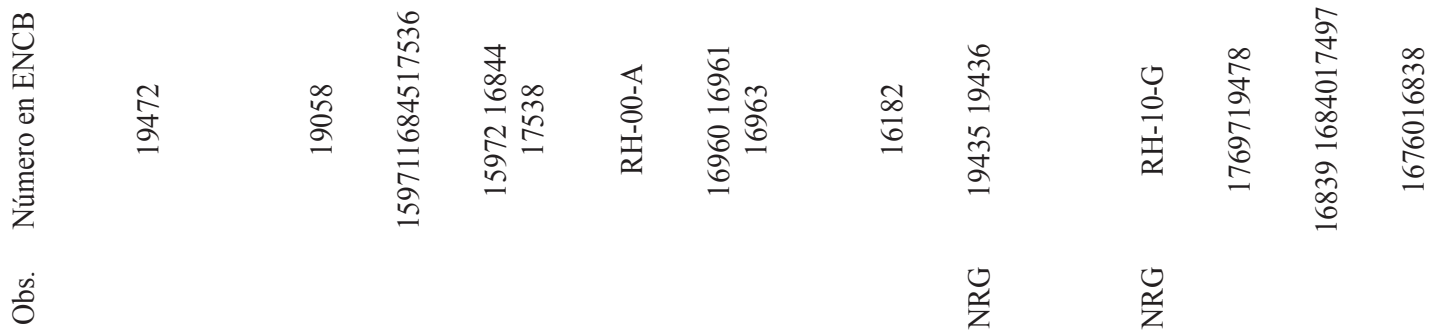

营

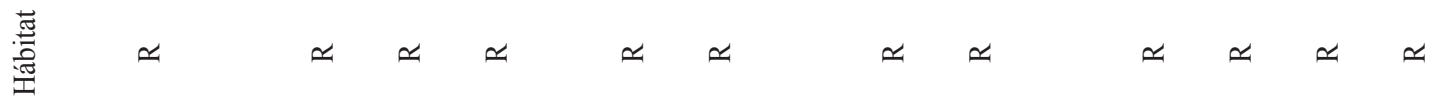

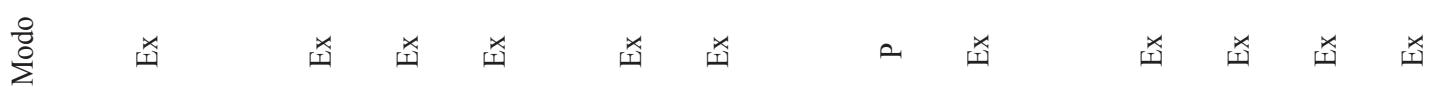

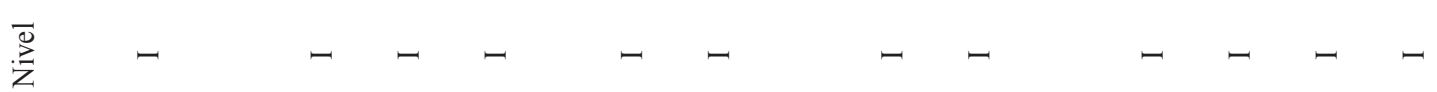

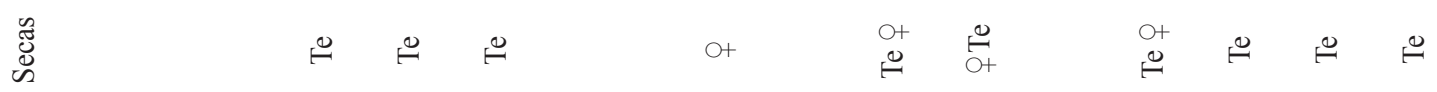

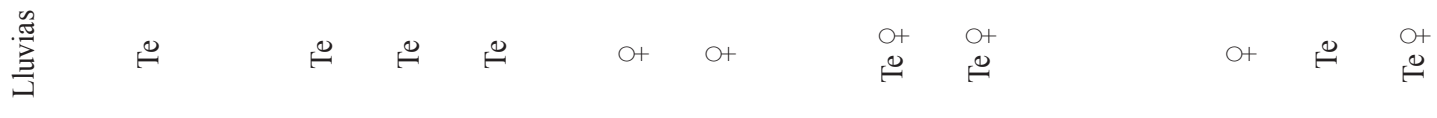

\begin{tabular}{|c|c|c|c|c|c|c|c|c|c|c|}
\hline$r$ & $\Xi \backsim$ & $\sim$ & $\Xi \backsim$ & $\sim$ & $\sim s$ & is & is & & & \\
\hline 6 ஏ & $\stackrel{\sim}{\Xi}$ & $\frac{\Omega}{\beth}$ & $\stackrel{\sim}{\beth}$ & $\stackrel{\sim}{\beth}$ & $\stackrel{\sim}{\Xi}$ & $\stackrel{\sim}{\Xi}$ & $\sim$ & $\sim$ & $\stackrel{\sim}{\Xi}$ & \\
\hline$n$ & & $\stackrel{\sim}{コ}$ & コ & コ & & & & & $\stackrel{\sim}{\Xi}$ & \\
\hline 寸 & & $\exists \backsim$ & $\Xi \backsim$ & コ & & & & & $\exists \backsim$ & コ \\
\hline$m$ & 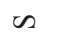 & $\Xi \backsim$ & $\Xi \backsim$ & $\Xi \backsim$ & & & & コ & & コ \\
\hline
\end{tabular}

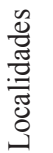

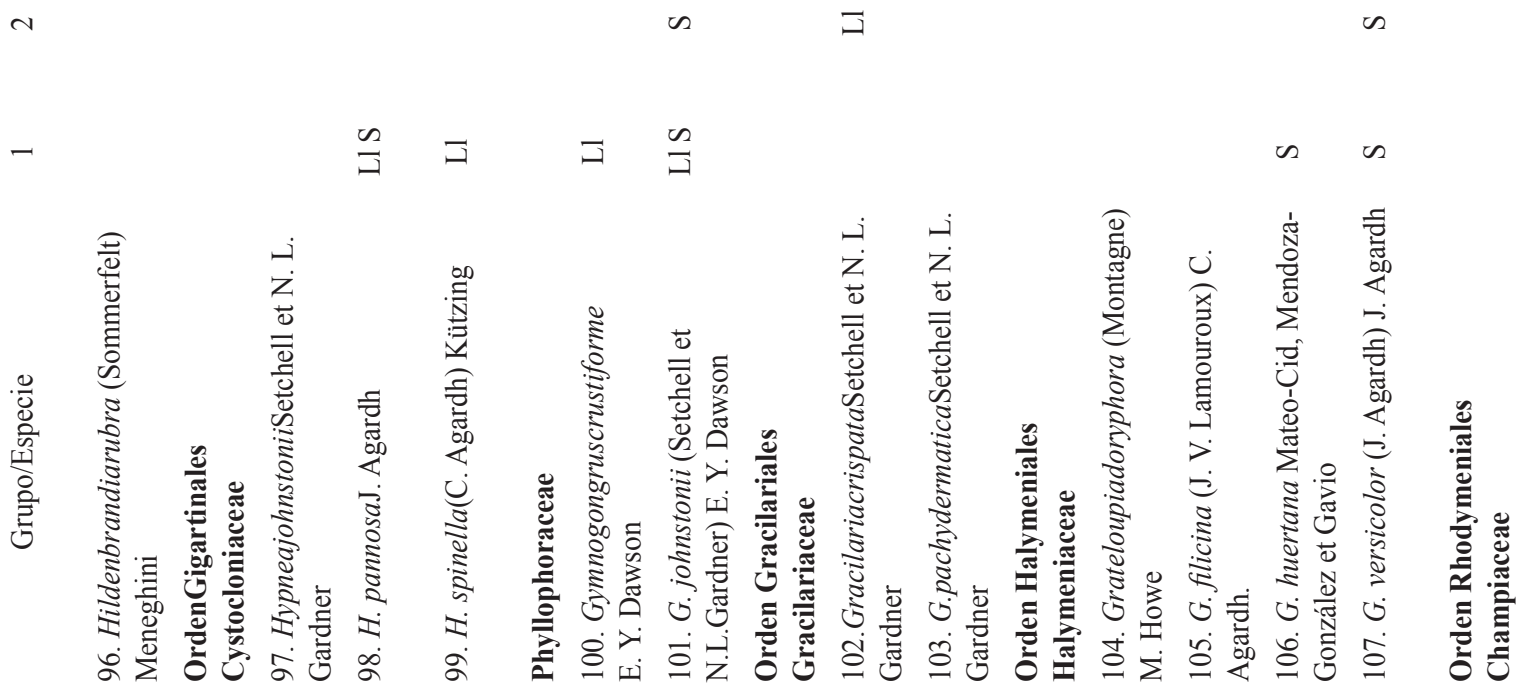




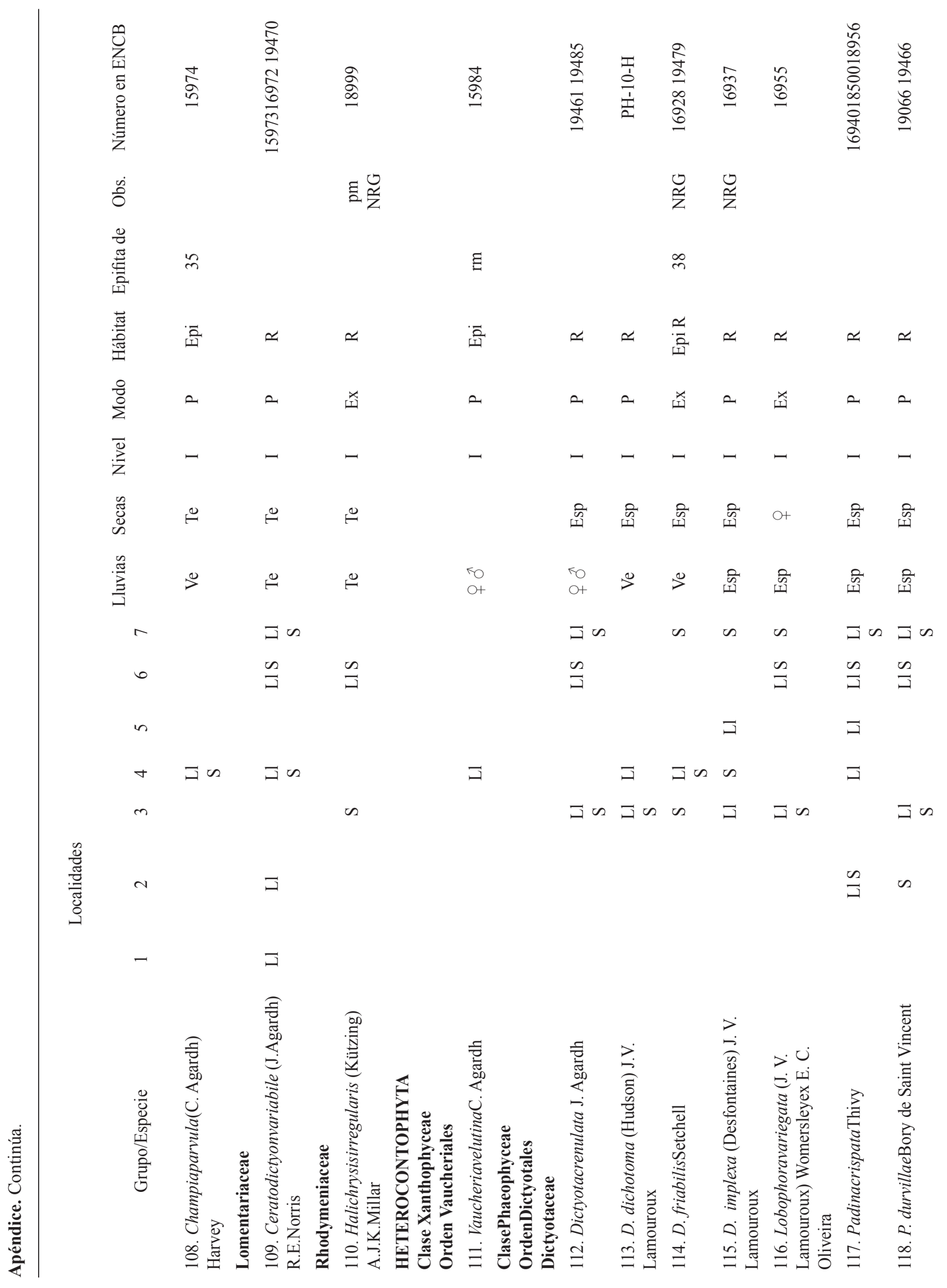




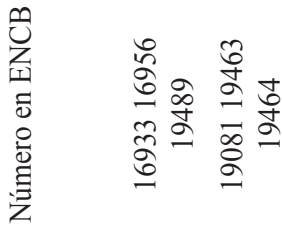

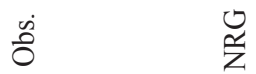

욜

营葍 $\simeq \simeq$

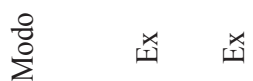

这 -

ठँ के के

苟 象

$$
\begin{array}{ll}
n & n \\
0 & \Xi \\
n & \Xi \infty \\
\infty & \Xi \infty
\end{array}
$$

离

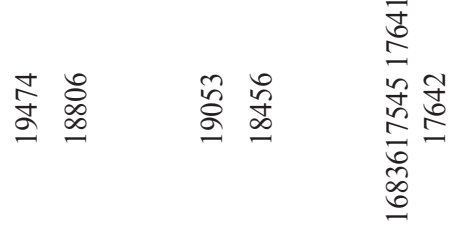

ฐ

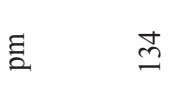

$\simeq \approx$

2.

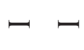

5

55

コ先

$\exists$

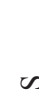

is

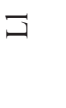

0 a $\quad \frac{a}{4}$

$\therefore 2 \quad \overrightarrow{0}$

コ

$\exists$ 的

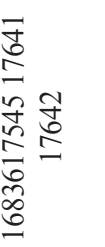

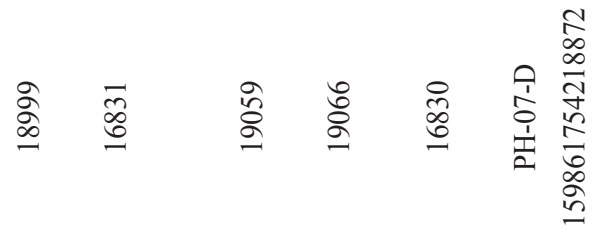

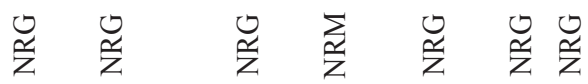

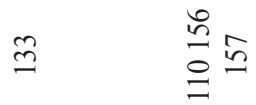

$\stackrel{\infty}{=} \stackrel{\infty}{n} \stackrel{\Xi}{=}$

๙

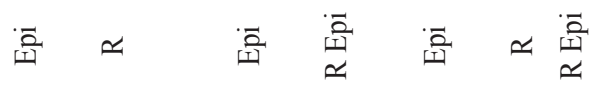

a a a a 肴 a

$\vec{z} \quad \frac{\vec{B}}{2}$

$\frac{\vec{\partial}}{2} \quad \frac{\vec{a}}{a} \quad \frac{\vec{a}}{a}$

$\frac{\overrightarrow{2}}{2}$

$\frac{7}{2}$

a

$\Xi$

in is

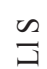

is

$\Xi$

is in

$\exists$ is

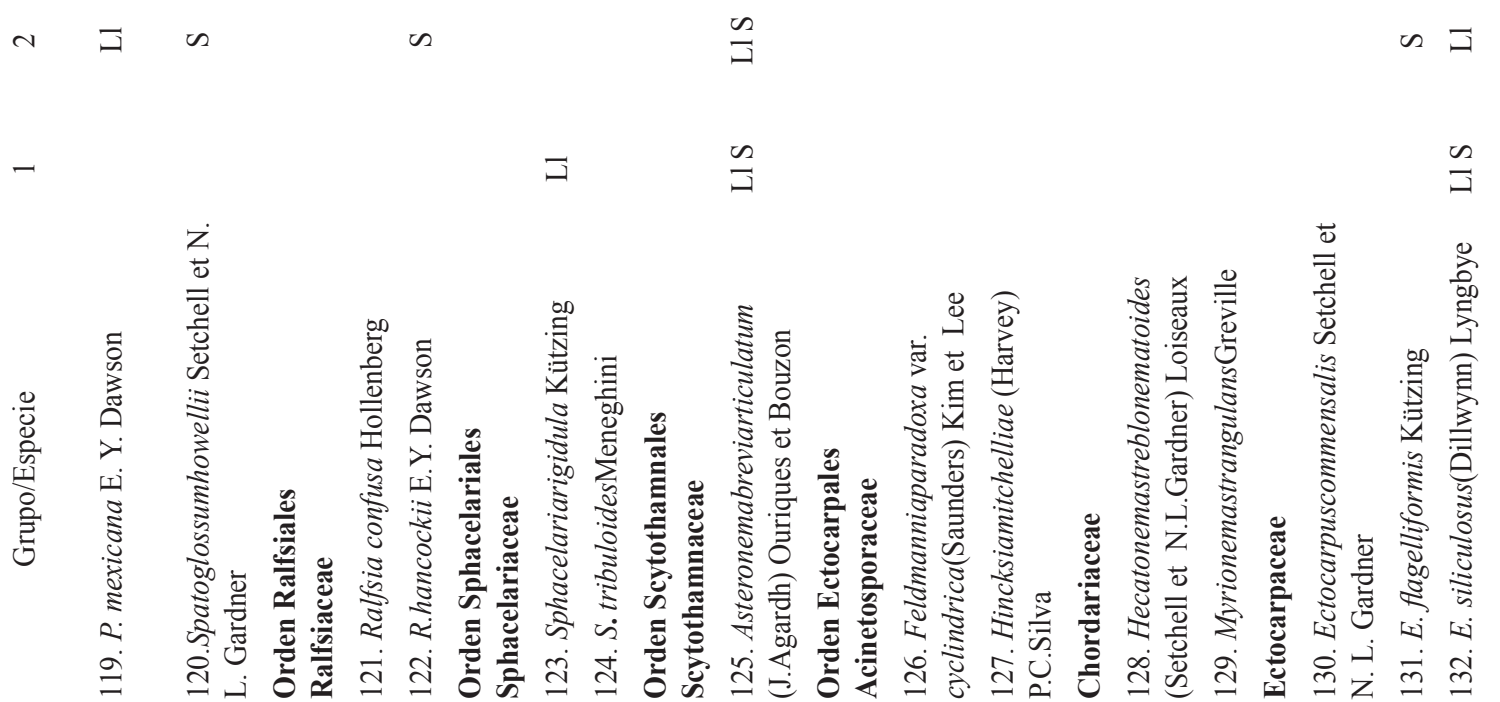

$\backsim \Xi$ 


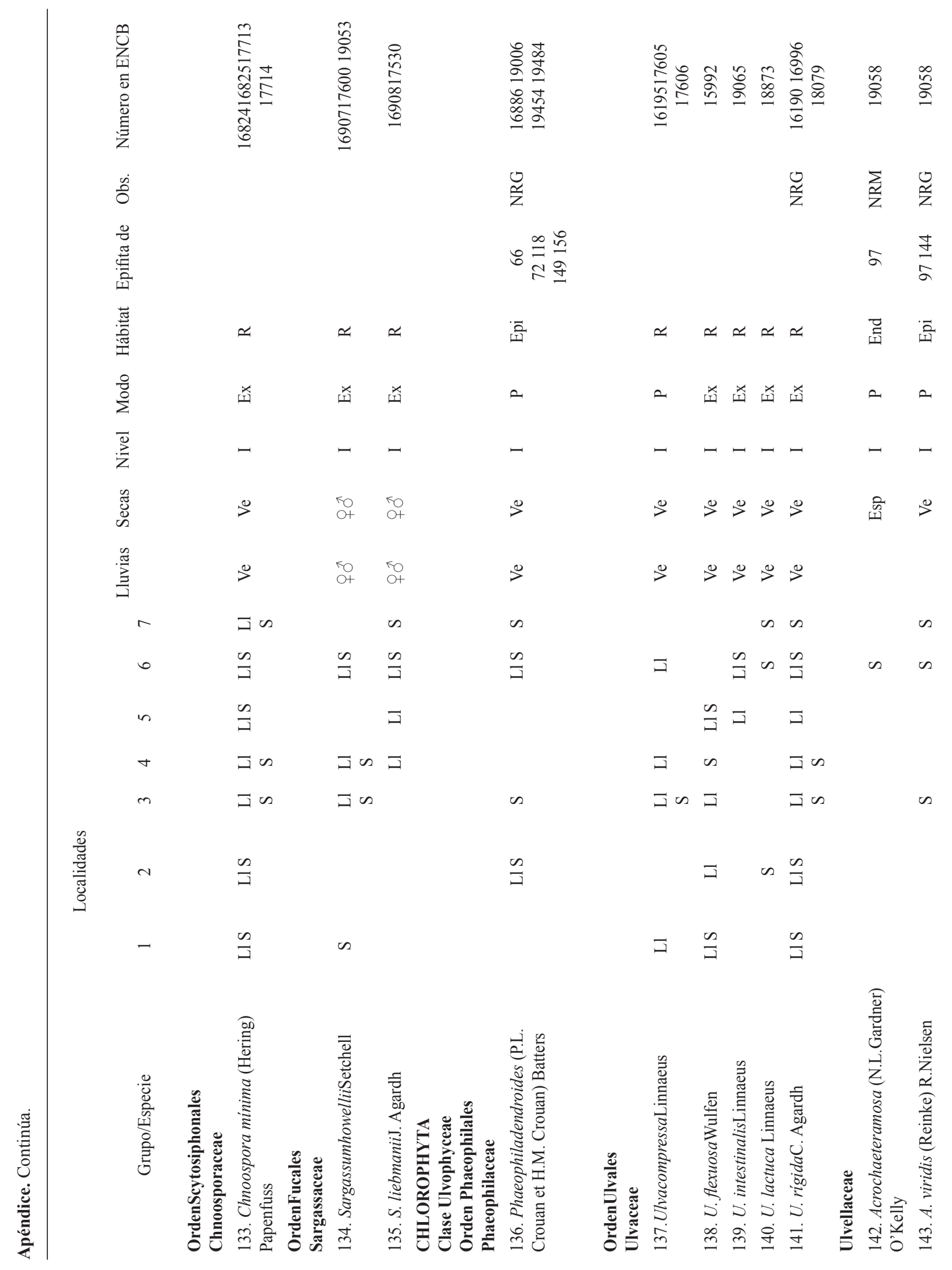




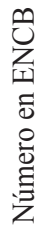

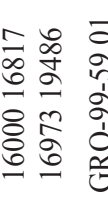

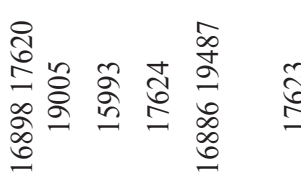

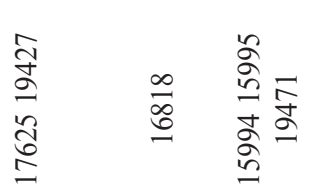

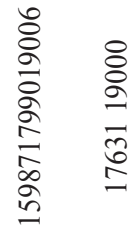

$\frac{n}{\infty}$

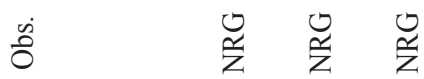

艺

宽

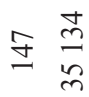

$\stackrel{\wp}{\curvearrowleft}$

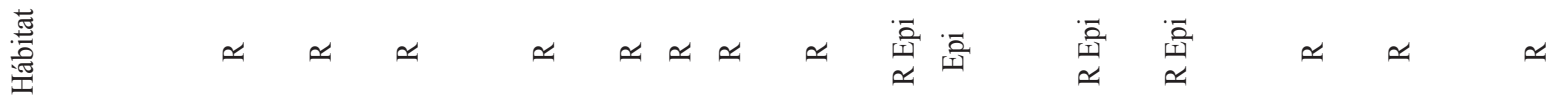

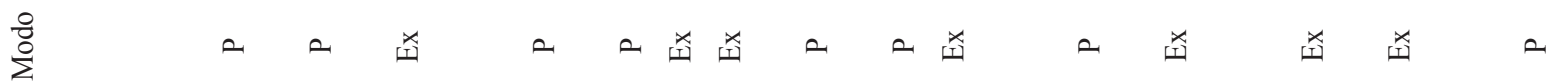

$\stackrel{\bar{z}}{2}$

๖ू

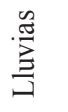

$>$ के

$>$

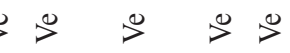

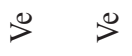

ํำ is is $>$

$>>>>$

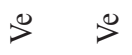

‡

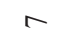

تn a Jun is

⿰ コ๐

o is is

a n en

$\stackrel{\sim}{\exists}$

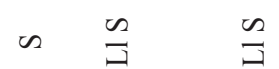

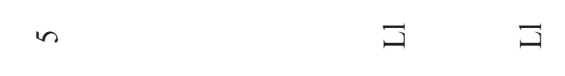

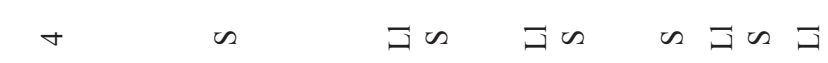

$\frac{0}{0}$

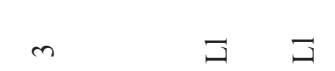

コのコ

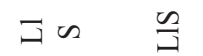

ヨッ ヨ

$\Xi$
ஏ

N

a

$\stackrel{\sim}{\Xi}$
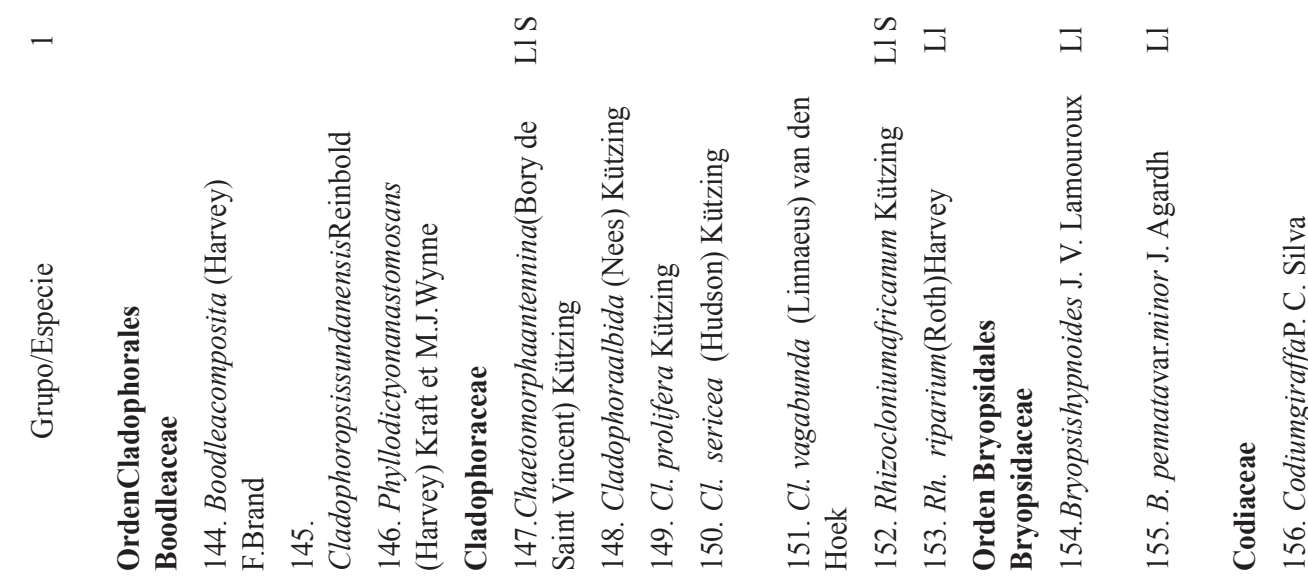

$\begin{array}{ll} & \\ & \end{array}$ 


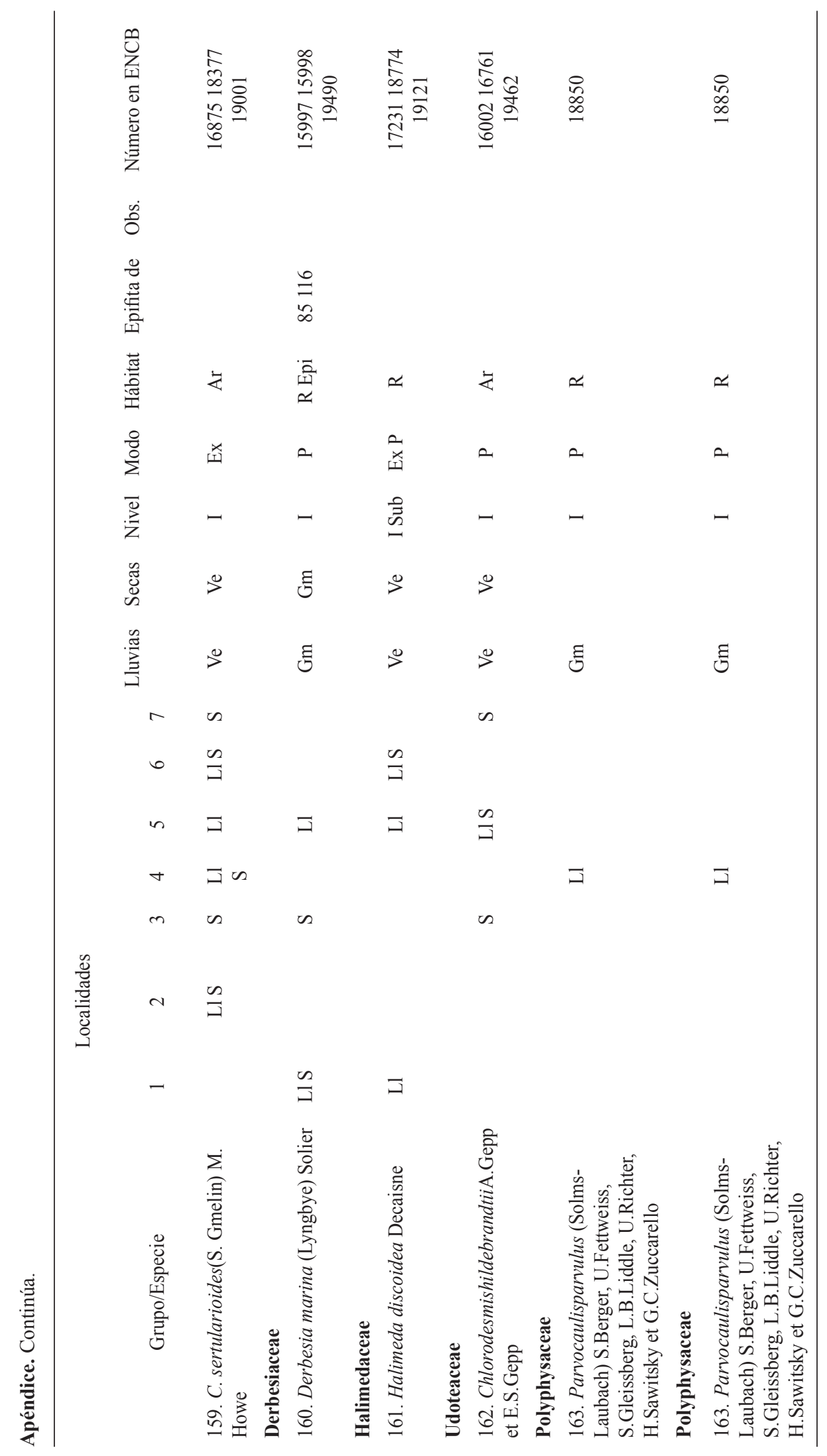

